TRANSACTIONS OF THE

AMERICAN MATHEMATICAL SOCIETY

Volume 350, Number 4, April 1998, Pages 1481-1518

S 0002-9947(98)02068-6

\title{
LIMIT THEOREMS FOR RANDOM TRANSFORMATIONS AND PROCESSES IN RANDOM ENVIRONMENTS
}

\author{
YURI KIFER
}

\begin{abstract}
I derive general relativized central limit theorems and laws of iterated logarithm for random transformations both via certain mixing assumptions and via the martingale differences approach. The results are applied to Markov chains in random environments, random subshifts of finite type, and random expanding in average transformations where I show that the conditions of the general theorems are satisfied and so the corresponding (fiberwise) central limit theorems and laws of iterated logarithm hold true in these cases. I consider also a continuous time version of such limit theorems for random suspensions which are continuous time random dynamical systems.
\end{abstract}

\section{INTRODUCTION}

Let $(\Omega, \mathcal{G}, P)$ be a probability space with an invertible $P$-preserving transformation $\theta: \Omega \rightarrow \Omega$, let $(\boldsymbol{\Xi}, \mathcal{F})$ be another measurable space, and let $\Xi$ be a measurable (with respect to the product $\mathcal{G} \times \mathcal{F}$ ) subset of $\boldsymbol{\Xi} \times \Omega$ with the "fibers" $\Xi^{\omega}=\{\xi \in \boldsymbol{\Xi}:(\xi, \omega) \in \Xi\} \in \mathcal{F}$. The set-up includes also a measurable map $\tau: \Xi \rightarrow \Xi$ such that $\tau(\xi, \omega)=\left(f_{\omega} \xi, \theta \omega\right)$ with $f_{\omega}: \Xi^{\omega} \rightarrow \Xi^{\theta \omega}$ being measurable "fiber" maps called random transformations (while $\tau$ is called the skew product transformation) with the composition rule $f_{\omega}^{n}=f_{\theta^{n-1} \omega} \circ \cdots \circ f_{\theta \omega} \circ f_{\omega}: \Xi^{\omega} \rightarrow \Xi^{\theta^{n} \omega}$.

Denote by $\mathcal{P}(\Xi)$ the space of probability measures $\mu$ on $\Xi$, and write $\mu \in \mathcal{P}_{P}(\Xi)$ if the marginal of $\mu$ on $\Omega$ is $P$. Such measures are determined by an essentially unique measurable family $\mu^{\omega}$ such that $\mu^{\omega}$ belongs to the space $\mathcal{P}\left(\Xi^{\omega}\right)$ of probability measures on $\Xi^{\omega}$ and

$$
\mu(d \xi, d \omega)=\mu^{\omega}(d \xi) P(d \omega) .
$$

It is easy to see that $\mu$ is $\tau$-invariant if and only if $f_{\omega} \mu^{\omega}=\mu^{\theta \omega}$.

There is already an extensive literature on the ergodic theory of random transformations, known also as the relativized ergodic theory, and, in particular, on the thermodynamic formalism (variational principle, equilibrium states, symbolic representations) for expanding random transformations and random subshifts of finite type (see [Ki1], [KK], [BG1], [BG2]) which yields also large deviations type results. In the deterministic case the study of the Ruelle-Perron-Frobenius (RPF) operator, which usually is the key point in the thermodynamic formalism approach, leads to estimates of decay of correlations which, in turn, via some probabilistic arguments yield central limit theorem (CLT) type results for corresponding dynamical systems

Received by the editors July 16, 1996.

1991 Mathematics Subject Classification. Primary 60F05; Secondary 58F15, 60J99, 60J05.

Key words and phrases. Central limit theorem, random transformations, random environment. Supported by the US-Israel Binational Science Foundation. 
(see [De]). Actually, the CLT requires much weaker conditions than one needs for the thermodynamic formalism set-up, but for many interesting classes of dynamical systems these conditions were verified only via RPF operator estimates.

In the random transformations framework the natural CLT has the following form. Let $\mu \in \mathcal{P}_{P}(\Xi)$ be $\tau$-invariant with the disintegration (1.1), and let $\varphi(\xi, \omega)=$ $\varphi_{\omega}(\xi), \xi \in \Xi^{\omega}$, be a measurable function. The aim of this paper is to provide conditions (which can be verified for specific cases) which ensure that if $\psi_{\omega}=$ $\varphi_{\omega}-\int \varphi_{\omega} d \mu^{\omega}$, then for $P$-almost all (a.a.) $\omega$ and any real $a$,

$$
\mu^{\omega}\left\{\xi \in \Xi^{\omega}: \frac{1}{\sqrt{n}} \sum_{i=0}^{n-1} \psi \circ \tau^{i}(\xi, \omega) \leq a\right\} \underset{n \rightarrow \infty}{\longrightarrow} \frac{1}{\sigma \sqrt{2 \pi}} \int_{-\infty}^{a} e^{\frac{x^{2}}{2 \sigma^{2}}} d x
$$

which may be called a relativized (or fiberwise) CLT or a CLT in random environments, depending on whether one takes a dynamical systems or a probabilistic point of view. I shall derive also a corresponding law of the iterated logarithm (LIL) saying that for $P-$ a.a. $\omega$ and $\mu^{\omega}$-a.a. $\xi$ the sum $\sum_{i=0}^{n-1} \psi \circ \tau^{i}(\xi, \omega)$ has the order $2 \sigma^{2} n \log \log \left(\sigma^{2} n\right)$.

The whole set-up can be reformulated in the language of processes in random environments. Namely, let $(\mathbf{X}, \mathcal{A})$ be another measurable space, $X$, a measurable subset of $\mathbf{X} \times \Omega$, and $Z: \Xi \rightarrow X$ a measurable "bundle" map, so that $Z(\xi, \omega)=$ $\left(Z^{\omega}(\xi), \omega\right)$, where $Z^{\omega}$ maps $\Xi^{\omega}$ to $X^{\omega} \in \mathcal{A}$. I view $\Omega$ as an environments space and $\Xi^{\omega}$ and $X^{\omega}$ as the path and the phase (or state) spaces, respectively, in the environment $\omega$ for the process $Z_{n}^{\omega}=Z \circ \tau^{n}(\xi, \omega)=Z^{\theta^{n} \omega}\left(f_{\omega}^{n} \xi\right)$. If $\mu \in \mathcal{P}_{P}(\Xi)$ is $\tau$-invariant, then $Z_{n}^{\omega}(\xi)$ can be considered as a usual stationary process on the probability space $(\Xi, \mathcal{G} \times \mathcal{F}, \mu)$, but my goal is to study properties of the process $Z_{n}^{\omega}(\xi)$ with respect to the measure $\mu^{\omega}$ for a fixed $P$-typical $\omega$, which usually requires different arguments. In particular, if there exists a measurable family of probability measures $P^{\omega}(x, \cdot)$ on $X^{\theta \omega}, x \in X^{\omega}$, such that for any measurable $\Gamma_{i} \subset X^{\theta^{i} \omega}, i=1, \ldots, n$,

$$
\begin{aligned}
& \mu^{\omega}\left\{\xi \in \Xi^{\omega}: Z_{1}^{\omega} \in \Gamma_{1}, Z_{2}^{\omega}(\xi) \in \Gamma_{2}, \ldots, Z_{n}^{\omega}(\xi) \in \Gamma_{n} \mid Z_{0}^{\omega}(\xi)=x\right\} \\
& =\int_{\Gamma_{1}} \ldots \int_{\Gamma_{n-1}} P^{\omega}\left(x, d y_{1}\right) \cdots P^{\theta^{n-2} \omega}\left(y_{n-2}, d y_{n-1}\right) P^{\theta^{n-1} \omega}\left(y_{n-1}, \Gamma_{n}\right),
\end{aligned}
$$

then $Z_{n}^{\omega}(\xi)$ becomes for each $\omega$ a time-inhomogenious Markov chain and the whole object is called a Markov chain, in a random environment. A particular case of this set-up when all $X^{\omega}$ coincide with one countable set was introduced as a model for stochastic automata, and its ergodic theory was studied in a number of papers (see $[\mathrm{Or}]$ and the references there). An $\omega$-wise CLT for this situation under rather indirect assumptions was obtained in [Co], but even for this particular case I shall derive here more general and more comprehensive results. I shall obtain first a CLT and an LIL in rather general circumstances, both under some uniform mixing assumptions and using the martingale differences approach. Then I shall verify the conditions of these theorems in the case of Markov chains in random environments under the random Doeblin condition introduced in [Ki3], and in the cases of random subshifts of finite type under certain random topological mixing conditions and random expanding in average transformations. A corresponding law of the iterated logorithm holds true for these cases, as well. Note that the large deviations type results were obtained for Markov chains in random environments in [Se] and [Ki3], and for random expanding transformations in [Ki1]. 
In the deterministic dynamics an important class of continuous time dynamical systems (flows) can be modelled by suspensions constructed by a well-mixing transformation and a Hölder continuous function. I introduce also the notion of random suspensions and obtain under natural conditions a CLT for this class of random continuous time dynamical systems.

\section{Preliminaries AND MAIN Results}

I shall start with the set-up which, includes a $\tau$-invariant probability measure $\mu$ on $\Xi$ with the disintegration (1.1) and a measurable family of $\sigma$-algebras $\mathcal{F}_{m, n}^{\omega}, n \geq$ $m, \omega \in \Omega$, of sets from $\Xi^{\omega}$ such that

$$
\mathcal{F}_{m, n}^{\omega} \subset \mathcal{F}_{m^{\prime}, n^{\prime}}^{\omega} \text { if } m^{\prime} \leq m \text { and } n^{\prime} \geq n \text { and } f_{\omega}^{-1} \mathcal{F}_{m, n}^{\theta \omega}=\mathcal{F}_{m+1, n+1}^{\omega} .
$$

The uniform mixing ( $\phi$-mixing) coefficient is defined by

$$
\phi_{i, j}^{\omega}=\sup _{A \in \mathcal{F}_{0, i}^{\omega}, \mu^{\omega}(A) \neq 0, B \in \mathcal{F}_{j, \infty}^{\omega}}\left|\frac{\mu^{\omega}(A \cap B)}{\mu^{\omega}(A)}-\mu^{\omega}(B)\right|, j>i .
$$

Let $\varphi=\varphi(\xi, \omega)=\varphi_{\omega}(\xi)$ be a measurable function on $\Xi$ so that $\varphi_{\omega}$ as a function on $\Xi^{\omega}$ is $\mathcal{F}_{0, \infty}^{\omega}$-measurable, and so in view of $(2.1), \varphi_{\theta^{i} \omega} \circ f_{\omega}^{i}$ is $\mathcal{F}_{i, \infty}^{\omega}$-measurable as a function on $\Xi^{\omega}$. The set-up includes also a measurable set $Q \subset \Omega$ with $P(Q)>0$ and the corresponding sequence of hitting times

$$
k_{i+1}(\omega)=\min \left\{k>k_{i}(\omega): \theta^{k} \omega \in Q\right\} \text { with } k_{0} \equiv 0 .
$$

Set

$$
\begin{gathered}
\psi_{\omega}=\varphi_{\omega}-E_{\mu^{\omega}} \varphi_{\omega}, \Psi(\xi, \omega)=\Psi_{\omega}(\xi)=\sum_{i=0}^{k_{1}(\omega)-1} \psi \circ \tau^{i}(\xi, \omega) \\
c(\omega)=\left(E_{\mu^{\omega}}\left|\psi_{\omega}\right|^{2}\right)^{1 / 2}, C(\omega)=\left(E_{\mu^{\omega}}\left|\Psi_{\omega}\right|^{2}\right)^{1 / 2} \\
d_{n}(\omega)=\left(E_{\mu^{\omega}}\left(\psi_{\omega}-E_{\mu^{\omega}}\left(\psi_{\omega} \mid \mathcal{F}_{0, n}^{\omega}\right)\right)^{2}\right)^{1 / 2} \\
D_{n}(\omega)=\left(E_{\mu^{\omega}}\left(\Psi_{\omega}-E_{\mu^{\omega}}\left(\Psi_{\omega} \mid \mathcal{F}_{0, n}^{\omega}\right)\right)^{2}\right)^{1 / 2}
\end{gathered}
$$

where $E_{\nu}$ always denotes the expectation (i.e. the integral) with respect to a probability measure $\nu$ and $E_{\nu}(\cdot \mid \cdot)$ is the corresponding conditional expectation. Observe that

$$
C(\omega) \leq \sum_{i=0}^{k_{1}(\omega)-1} c\left(\theta^{i} \omega\right) \text { and } D_{n}(\omega) \leq \sum_{i=0}^{k_{1}(\omega)-1} d_{n-i}\left(\theta^{i} \omega\right)
$$

Set $\Theta=\theta^{k_{1}(\omega)}, F_{\omega}=f_{\omega}^{k_{1}(\omega)}$, and $T(\xi, \omega)=\left(F_{\omega} \xi, \Theta \omega\right)$. I always assume that $\theta:(\Omega, P) \longrightarrow(\Omega, P)$ is ergodic; then it is well known (see, for instance, [CFS]) that $\Theta$ is an ergodic measure-preserving transformation on the space $\left(Q, P_{Q}\right)$, where $P_{Q}(A)=\frac{P(A \cap Q)}{P(Q)}$. I shall denote by $\Xi_{Q}$ the restriction of $\Xi$ to $\Xi \times Q$ and by $\mu_{Q}$ the normalized restriction of $\mu$ to $\Xi_{Q}$, i.e. $d \mu_{Q}(\xi, \omega)=d \mu^{\omega}(\xi) d P_{Q}(\omega)$. It follows that $\mu_{Q}$ is invariant under the action of $T$. 
2.1. Theorem. Let $\phi_{j}=\sup _{\omega, i \geq 1} \phi_{k_{i}(\omega), k_{i+j}(\omega)}^{\omega}$ and $\beta_{j}=\left(E_{P_{Q}} D_{k_{j}}^{2}\right)^{1 / 2}$, and suppose that

$$
\begin{gathered}
\sum_{j=1}^{\infty} \phi_{j}^{1 / 2}<\infty \\
\sum_{j=1}^{\infty} \beta_{j}<\infty
\end{gathered}
$$

and

$$
E_{P_{Q}}\left(\sum_{i=0}^{k_{1}-1} c \circ \theta^{i}\right)^{2}<\infty .
$$

Then P-a.s.(almost surely),

$$
\sigma^{2} \stackrel{\text { def }}{=} \lim _{n \rightarrow \infty} \frac{1}{n} E_{\mu^{\omega}}\left(\sum_{j=0}^{n-1} \psi_{\theta^{j} \omega} \circ f_{\omega}^{j}\right)^{2}=P(Q)\left(E_{\mu_{Q}} \Psi^{2}+\sum_{l=1}^{\infty} E_{\mu_{Q}}\left(\Psi\left(\Psi \circ T^{l}\right)\right)\right)
$$

and the series in the right hand side of (2.8) converges. Furthermore, P-a.s. for any number $a$,

$$
\lim _{n \rightarrow \infty} \mu^{\omega}\left\{\xi \in \Xi^{\omega}: \frac{1}{\sqrt{n}} \sum_{i=0}^{n-1} \psi \circ \tau^{i}(\xi, \omega) \leq a\right\}=\frac{1}{\sigma \sqrt{2 \pi}} \int_{-\infty}^{a} e^{-\frac{x^{2}}{2 \sigma^{2}}} d x,
$$

i.e. for $P$-a.a.w the $\mu^{\omega}$-distribution of $n^{-1 / 2} \sum_{i=0}^{n-1}\left(\psi \circ \tau^{i}\right)_{\omega}$ converges to the normal distribution with zero mean and variance $\sigma^{2}$, which in case $\sigma=0$ is understood as the unit mass at 0 . Finally, $\sigma=0$ if and only if there exists a function $\eta$ on $\Xi_{Q}$ from $L^{2}\left(\Xi_{Q}, \mu_{Q}\right)$ such that $\mu_{Q}-$ a.s.,

$$
\Psi \circ T=\eta \circ T-\eta
$$

Moreover, assuming that $\sigma>0$, the following invariance principle for the law of the iterated logarithm (LIL) holds true. If $\zeta(t)=(2 t \log \log t)^{1 / 2}$ and

$$
\begin{aligned}
& \eta_{n}(t)=\left(\zeta\left(\sigma^{2} n\right)\right)^{-1} \sum_{j=0}^{k-1}\left(\psi \circ \tau^{j}+(n t-k) \psi \circ \tau^{k}\right) \\
& \qquad \text { for } t \in\left[\frac{k}{n}, \frac{k+1}{n}\right), k=0,1, \ldots, n-1,
\end{aligned}
$$

then $\mu_{Q}-$ a.s. the sequence of functions $\left\{\eta_{n}(\cdot), n \geq 3 / \sigma^{2}\right\}$ is relatively compact in the space $C[0,1]$ (of continuous functions on $[0,1]$ considered with the supremum norm), and the set of its limit points as $n \rightarrow \infty$ coincides with the set $K$ of absolutely continuous $x \in C[0,1]$ with $\int_{0}^{1}(\dot{x}(t))^{2} d t \leq 1$.

I note that though there are some results on CLT for nonstationary processes (see, for instance, [VR]) which at first sight could be used in Theorem 2.1, it turns out that the assumptions needed for these results are not satisfied for the interesting models I have in mind. Namely, [VR] requires that

$$
(n-m)^{-1} E_{\mu^{\omega}} \sum_{m \leq j \leq n-1} \Psi^{2} \circ T^{i}
$$


is uniformly in $n$ and $m$ bounded away from zero and infinity, which may be satisfied only when $k_{1}$ is uniformly bounded - a very restrictive precondition. Thus in order to derive Theorem 2.1 one has to take into account in an essential way the stationary evolution on the space $(\Omega, P)$. My proof of Theorem 2.1 borrows few elements from [Co], where a partial case of Markov measures $\mu^{\omega}$ (with a countable phase space of the corresponding Markov chain) and functions $\varphi_{\omega}$ measurable with respect to $\mathcal{F}_{0,0}^{\omega}$ was considered. The extension to a class of functions $\varphi_{\omega}$ more general than in [Co] follows by a slight modification of arguments from Section 20 of [Bi1]. The LIL part of Theorem 2.1 follows via a random martingale differences representation, which provides also another proof of the CLT part of Theorem 2.1. After this paper was accepted B.-Z. Rubshtein showed me [Ru] where CLT was proved in a similar setup using martingale differences under rather general conditions.

It is much more convenient to formulate the assumptions of Theorem 2.1 and of other results of this section in terms of the function $\Psi$ and not in terms of the original function $\varphi$ and the set $Q$, but it is useful to have also some sufficient conditions on $\varphi$ and on $k_{1}$ which yield these assumptions. I shall now formulate a sufficient condition of this type (which will be proved at the end of Section 3) in terms of mixing and approximations with respect to a family of $\sigma$-algebras $\mathcal{G}_{m, n} \subset \mathcal{G},-\infty \leq m \leq n \leq \infty$, on $\Omega$ such that $\mathcal{G}_{m . n} \subset \mathcal{G}_{m^{\prime}, n^{\prime}}$ if $m^{\prime} \leq m, n^{\prime} \geq n$ and $\theta \mathcal{G}_{m, n}=\mathcal{G}_{m-1, n-1}$. The strong mixing $(\alpha-$ mixing) coefficient with respect to this family of $\sigma$-algebras is defined by

$$
\alpha_{n}=\sup _{k, A \in \mathcal{G}_{-\infty, k}, B \in \mathcal{G}_{k+n, \infty}}|P(A \cap B)-P(A) P(B)| .
$$

Set also

$$
\gamma_{n}=E_{P}\left|\mathbb{I}_{Q}-E_{P}\left(\mathbb{I}_{Q} \mid \mathcal{G}_{-n, n}\right)\right|,
$$

where $\mathbb{I}_{Q}(\omega)=1$ if $\omega \in Q$ and $=0$, otherwise.

2.2. Proposition. Suppose that

$\sup _{\omega} c(\omega)<\infty$ and $\sum_{l=1}^{\infty} l^{2}\left(\alpha_{l}+\gamma_{l}\right)<\infty$ or $E_{P_{Q}} c^{4}<\infty$ and $\sum_{l=1}^{\infty} l^{5 / 4}\left(\alpha_{l}+\gamma_{l}\right)^{1 / 4}<\infty$.

Then (2.7) holds true. If $Q=Q_{L}=\left\{\omega: L_{\omega} \leq L\right\}$ for some random variable $L_{\omega}$ on $(\Omega, P)$ and a constant $L$, then $\gamma_{n} \leq 2 \tilde{\gamma}_{n}$ with $\tilde{\gamma}_{n}=E_{P}\left|L_{\omega}-E_{P}\left(L_{\omega} \mid \mathcal{G}_{-n, n}\right)\right|$, and so one can write $\tilde{\gamma}_{l}$ in place of $\gamma_{l}$ in (2.13), which provides a sufficient condition directly in terms of $L_{\omega}$, and the latter has an explicit representation in the specific cases of Theorems 2.4-2.6 below.

Next, I shall describe another approach which sometimes is more convenient since it does not require an explicit uniform mixing condition with respect to a certain family of $\sigma$-algebras as in Theorem 2.1. The set-up includes the same objects $f_{\omega}, \theta, \tau, \varphi, \psi, \mu, Q, k_{i}, \Psi, F_{\omega}, \Theta, T, P_{Q}, \Xi_{Q}$, and $\mu_{Q}$ as above, but now in place of a family of $\sigma$-fields $\mathcal{F}_{m, n}^{\omega}$ I consider another family of $\sigma$-algebras $\mathcal{T}_{l}^{\omega}=$ $\left(f_{\omega}^{l}\right)^{-1} \mathcal{T}_{0}^{\theta^{l} \omega}$, where $\mathcal{T}_{0}^{\omega}$ for each $\omega$ is the restriction of the $\sigma$-algebra $\mathcal{F}$ to $\Xi^{\omega} \subset$ $\boldsymbol{\Xi}$. Then $\mathcal{T}_{l}^{\omega}, l=0,1, \ldots$, is a nonincreasing sequence of $\sigma$-algebras on $\Xi^{\omega}$. Let 
$u_{\omega}: L^{2}\left(\Xi^{\theta \omega}, \mu^{\theta \omega}\right) \longrightarrow L^{2}\left(\Xi^{\omega}, \mu^{\omega}\right)$ be an isometry operator acting by the formula $u_{\omega} \varphi(\xi)=\varphi\left(f_{\omega} \xi\right)$ and let $u_{\omega}^{*}: L^{2}\left(\Xi^{\omega}, \mu^{\omega}\right) \longrightarrow L^{2}\left(\Xi^{\theta \omega}, \mu^{\theta \omega}\right)$ be its conjugate defined by $\int \varphi\left(u_{\omega}^{*} \tilde{\varphi}\right) d \mu^{\theta \omega}=\int\left(u_{\omega} \varphi\right) \tilde{\varphi} d \mu^{\omega}$ for any $\varphi \in L^{2}\left(\Xi^{\theta \omega}, \mu^{\theta \omega}\right)$ and $\tilde{\varphi} \in L^{2}\left(\Xi^{\omega}, \mu^{\omega}\right)$. It is easy to see that $u_{\omega} u_{\omega}^{*}: L^{2}\left(\Xi^{\omega}, \mu^{\omega}\right) \longrightarrow L^{2}\left(\Xi^{\omega}, \mu^{\omega}\right)$ is the orthogonal projection to $u_{\omega} L^{2}\left(\Xi^{\theta \omega}, \mu^{\theta \omega}\right)$, and the last set is exactly the set of $\mathcal{T}_{1}^{\omega}$-measurable functions in $L^{2}\left(\Xi^{\omega}, \mu^{\omega}\right)$. Introduce also the operator $U_{\omega}=u_{\omega}^{k_{1}(\omega)}$, where $u_{\omega}^{n}=u_{\theta^{n-1} \omega} \circ \cdots \circ u_{\theta \omega} \circ u_{\omega}$, and let $U_{\omega}^{*}$ be its conjugate. The proof of the following result emplois nonstationary backwards martingale differences apporoximations and proceeds along the scheme similar to [Li], which, in turn, is a modification of [Go], though both papers dealt with the stationary case only.

2.3. Theorem. Assume that (2.7) holds true and that the following two conditions are satisfied:

$$
E_{P_{Q}} \sum_{n=0}^{\infty}\left|E_{\mu^{\omega}}\left(\Psi_{\omega}\left(\Psi \circ T^{n}\right)_{\omega}\right)\right|<\infty
$$

and

$$
E_{P_{Q}} E_{\mu^{\omega}}\left(\sum_{n=0}^{\infty}\left|U_{\Theta^{-n} \omega}^{* n} \Psi_{\Theta^{-n} \omega}\right|\right)^{2}<\infty,
$$

where $U_{\omega}^{* n}=U_{\Theta^{n-1} \omega}^{*} \circ \cdots \circ U_{\Theta \omega}^{*} U_{\omega}^{*}$. Then $P$-a.s. (2.8) and (2.9) hold true, and the criterion (2.10) for $\sigma=0$ remains valid, as well. Moreover, assuming that $\sigma>0$, the invariance principle for the law of iterated logarithm (LIL) stated in Theorem 2.1 holds true.

Actually, by an approximation argument similar to [Li] one can prove Theorem 2.3 assuming the weaker condition

$$
E_{P_{Q}}\left(\sum_{n=0}^{\infty} E_{\mu^{\omega}}\left|U_{\Theta^{-n} \omega}^{* n} \Psi_{\Theta^{-n} \omega}\right|\right)^{2}<\infty
$$

in place of (ii), but since for the models I have in mind the verification of (ii') is not easier than that of (ii) and since, on the other hand, the proof of Theorem 2.3 under ( $\left.\mathrm{ii}^{\prime}\right)$ is more complicated, I restrict myself to the case when (ii) holds true.

Next, I shall discuss specific examples of processes in random environments and random transformations for which either the mixing conditions of Theorem 2.1 or the convergence conditions of Theorem 2.3 needed for martingale differences approximations hold true. First, I shall consider the case when the $\mu^{\omega}$ 's are Markov measures, and using Theorem 2.1 I shall obtain a result which both generalizes and specifies the CLT for Markov chains in random environments from [Co], deriving it for a more general class of functions and showing that the uniform mixing condition required in Theorem 2.1 follows if one assumes the random Doeblin condition for transition probabilities from [Ki3]. Thus, assume that $\mu^{\omega}$ is given by (1.3), but in place of the conditional probabilities there I write

$$
\begin{aligned}
& \mu^{\omega}\left\{\xi \in \Xi^{\omega}: Z_{1}^{\omega}(\xi) \in \Gamma_{1}, Z_{2}^{\omega}(\xi) \in \Gamma_{2}, \ldots, Z_{n}^{\omega}(\xi) \in \Gamma_{n}\right\} \\
& =\int_{X^{\omega}} \eta^{\omega}(d x) \int_{\Gamma_{1}} \ldots \int_{\Gamma_{n-1}} P^{\omega}\left(x, d y_{1}\right) \cdots P^{\theta^{n-2} \omega}\left(y_{n-2}, d y_{n-1}\right) P^{\theta^{n-1} \omega}\left(y_{n-1}, \Gamma_{n}\right),
\end{aligned}
$$


where $X^{\omega}, P^{\omega}(x, \cdot)$, and $\Gamma_{i}$ are the same as in (1.3) and $\eta^{\omega}, \omega \in \Omega$, is a measurable family of probability measures on $X^{\omega}$ such that for any measurable $\Gamma \subset X^{\omega}$,

$$
\int_{X^{\omega}} \eta^{\omega}(d x) P^{\omega}(x, \Gamma)=\eta^{\theta \omega}(\Gamma)
$$

Let

$$
P^{\omega}(n, x, \Gamma)=\int_{X^{\theta \omega}} \cdots \int_{X^{\theta^{n-1} \omega}} P^{\omega}\left(x, d y_{1}\right) P^{\theta \omega}\left(y_{1}, d y_{2}\right) \cdots P^{\theta^{n-1} \omega}\left(y_{n-1}, \Gamma\right)
$$

and assume that there exist random variables $N=N_{\omega} \in \mathbb{Z}_{+}=\{1,2, \ldots\}$ and $\gamma_{\omega}>0$ and a measurable family of measures $m^{\omega} \in \mathcal{P}\left(X^{\omega}\right)$ such that for $P$-a.a. $\omega \in \Omega$, any $x \in X^{\theta^{-N} \omega}$, and each measurable $\Gamma \subset X^{\omega}$,

$$
P^{\theta^{-N} \omega}(N, x, \Gamma) \geq \gamma_{\omega} m^{\omega}(\Gamma),
$$

which is a randomized version of the Doeblin condition introduced in [Ki3]. It follows from [Ki3] that (2.16) implies the existence and uniqueness of a measure $\eta^{\omega}$ satisfying (2.15). Let $\sigma$-algebras $\mathcal{F}_{m, n}^{\omega}$ be generated by all sets of the form $\left\{\xi: Z_{l}^{\omega}(\xi) \in \Gamma\right\}, l=m, m+1, \ldots, n$, for measurable $\Gamma \subset X^{\theta^{l} \omega}$.

2.4. Theorem. Let $Q=Q_{L}=\left\{\omega: \max \left(N_{\omega}, \gamma_{\omega}^{-1}\right) \leq L\right\}$ for a sufficiently large $L$, so that $P(Q)>0$. Suppose that (2.16) is satisfied, $\mu^{\omega}$ is defined by (2.14), and the $k_{i}(\omega)$ 's are defined by (2.3). Then the condition (2.5) holds true. Thus if $\varphi$ is a measurable function on $\Xi$ as in Theorem 2.1 satisfying the conditions (2.6) and (2.7), then (2.8) and (2.9) hold true, as well. If $\varphi_{\omega}(\xi)=\varphi_{\omega}\left(Z^{\omega}(\xi)\right)$ is, in fact, a function on $X$ (and, as before, $\psi_{\omega}=\varphi_{\omega}-E_{\mu^{\omega}} \varphi_{\omega}$ ), then (2.9) can be written in the form

$$
\begin{aligned}
& \lim _{n \rightarrow \infty} \mu^{\omega}\left\{\xi \in \Xi^{\omega}: \frac{1}{\sqrt{n}} \sum_{k=0}^{n-1} \psi_{\theta^{k} \omega}\left(Z_{k}^{\omega}(\xi)\right) \leq a \mid Z_{0}^{\omega}(\xi)=x\right\} \\
& =\lim _{n \rightarrow \infty} \mu^{\omega}\left\{\xi \in \Xi^{\omega}: \frac{1}{\sqrt{n}} \sum_{k=0}^{n-1} \psi_{\theta^{k} \omega}\left(Z_{k}^{\omega}(\xi)\right) \leq a\right\}=\frac{1}{\sigma \sqrt{2 \pi}} \int_{-\infty}^{a} e^{-\frac{u^{2}}{2 \sigma^{2}}} d u,
\end{aligned}
$$

which is satisfied for any initial point $x \in X^{\omega}$. A corresponding LIL described in Theorem 2.1 holds true for this case, as well.

Next, I shall deal with random subshifts of finite type (see [BG2] and [KK]). In this case $\Xi^{\omega}=\left\{\xi=\left(\xi_{0}, \xi_{1}, \ldots\right): \xi_{i} \in\left\{1, \ldots, \ell\left(\theta^{i} \omega\right)\right\}\right.$ and $a_{\xi_{i} \xi_{i+1}}\left(\theta^{i} \omega\right)=1$ for all $i=0,1, \ldots\}$, where $\ell=\ell(\omega)$, is a random variable on $(\Omega, P)$ satisfying

$$
\int \log \ell d P<\infty
$$

and $A(\omega)=\left(a_{i j}(\omega)\right), \omega \in \Omega$ is a measurable family of $\ell(\omega) \times \ell(\theta \omega)$-matrices with 0 and 1 entries such that $P$-a.s. $A(\omega)$ has no zero row. A random subshift of finite type is called topologically mixing if there exists a $\mathbb{Z}^{+}=\{1,2, \ldots\}$ - valued random variable $\tilde{N}=\tilde{N}_{\omega}<\infty$ on $(\Omega, \mathcal{G}, P)$ so that $A\left(\theta^{\tilde{N}} \omega\right) \cdots A\left(\theta^{-2} \omega\right) A\left(\theta^{-1} \omega\right)$ is a matrix with positive entries for $P$-a.a. $\omega$. This property is equivalent to existence of random variables $N=N_{\omega}<\infty$ and $\tilde{N}=\tilde{N}_{\omega}<\infty$ such that for any $n \geq N_{\omega}$ and $k \geq \tilde{N}_{\omega}$ the matrices $A(\omega) A(\theta \omega) \cdots A\left(\theta^{n} \omega\right)$ and $A\left(\theta^{-k} \omega\right) A\left(\theta^{-k+1} \omega\right) \cdots A\left(\theta^{-1} \omega\right)$ have only positive entries. The maps $f_{\omega}$ act by shifts, i.e. $\left(f_{\omega} \xi\right)_{j}=\xi_{j+1}$ for each $\xi \in \Xi^{\omega}$. The spaces $\Xi^{\omega}$ are imbedded into the compact space $\Xi=\overline{\mathbb{Z}}^{+} \times \overline{\mathbb{Z}}^{+} \times \cdots$, 
which is the infinite product of the one-point compactifications $\overline{\mathbb{Z}}^{+}=\mathbb{Z}^{+} \cup\{\infty\}$ of $\mathbb{Z}^{+}$with a metric on $\Xi$ given by

$$
d(\xi, \tilde{\xi})=\sum_{i=0}^{\infty} 2^{-i}\left|\frac{1}{\xi_{i}}-\frac{1}{\tilde{\xi}_{i}}\right| .
$$

For any measurable function $g=g(\xi, \omega)=g_{\omega}(\xi)$ on $\Xi \times \Omega$ set

$$
\operatorname{var}_{n} g_{\omega}=\sup \left\{\left|g_{\omega}(\xi)-g_{\omega}(\tilde{\xi})\right|: \xi_{i}=\tilde{\xi}_{i} \forall i=0,1, \ldots, n-1\right\} .
$$

Consider $g$ such that there exist $\kappa>0$ and a random variable $K_{g}(\omega)>0$ satisfying

$$
E_{P}\left|\log K_{g}(\omega)\right|<\infty
$$

and, for any $n=0,1,2, \ldots$,

$$
\operatorname{var}_{n} g_{\omega} \leq K_{g}(\omega) e^{-\kappa n}
$$

Suppose also that

$$
E_{P} \sup _{\xi}\left|g_{\omega}(\xi)\right|<\infty .
$$

The random Ruelle-Perron-Frobenius (RPF) operator $\mathcal{L}_{\gamma}^{\omega}$ corresponding to a function $g$ maps functions on $\Xi^{\omega}$ to functions on $\Xi^{\theta \omega}$ by the formula

$$
\mathcal{L}_{g}^{\omega} q(\xi)=\sum_{\zeta \in f_{\omega}^{-1} \xi} e^{g_{\omega}(\zeta)} q(\zeta)
$$

If $g$ satisfies (2.21)-(2.23), then the random RPF theorem for a topologically mixing random subshift of finite type yields (see $[\mathrm{KK}]$ and in a more restricted form [BG2]) that there exist a unique positive random variable $\lambda=\lambda_{\omega}$ with $E_{P}|\log \lambda|<\infty$, a positive measurable in $(\xi, \omega)$ and continuous in $\xi$ function $h=h(\xi, \omega)=h_{\omega}(\xi)$, and probability measures $\nu^{\omega}$ on $\Xi^{\omega}$ such that

$$
\mathcal{L}_{g}^{\omega} h_{\omega}=\lambda_{\omega} h_{\theta \omega},\left(\mathcal{L}_{g}^{\omega}\right)^{*} \nu^{\theta \omega}=\lambda_{\omega} \nu^{\omega}, \text { and } \int_{\Xi \omega} h_{\omega} d \nu^{\omega}=1 .
$$

Then probability measures $\mu^{\omega}$ (called random Gibbs measures) such that

$$
\frac{d \mu^{\omega}}{d \nu^{\omega}}(\xi)=h_{\omega}(\xi)
$$

satisfy

$$
f_{\omega} \mu^{\omega}=\mu^{\theta \omega},
$$

and the corresponding measure $\mu$ with the disintegration (1.1) is invariant with respect to the corresponding skew product transformation $\tau$.

Let $\mathcal{F}_{m, n}^{\omega}, n<\infty$, be the finite $\sigma$-algebra generated by cylinder sets

$$
C_{\eta_{m}, \eta_{m+1}, \ldots, \eta_{n}}=\left\{\xi=\left(\xi_{0}, \xi_{1}, \ldots\right): \xi_{i}=\eta_{i} \text { for } i=m, m+1, \ldots, n\right\},
$$

and let $\mathcal{F}_{m, \infty}^{\omega}$ be the minimal $\sigma$-algebra containing $\bigcup_{n \geq m} \mathcal{F}_{m, n}^{\omega}$.

2.5. Theorem. Suppose that a random subshift of finite type is topologically mixing, (2.18) is satisfied, the $\sigma$-algebras $\mathcal{F}_{m, n}^{\omega}$ are defined as above, and a measurable family of probability measures $\mu^{\omega}$ is constructed by a function $g$ satisfying (2.21)(2.23) so that (2.25)-(2.27) hold true. Then one can choose a set $Q$ in the form $Q=Q_{L}=\left\{\omega: L_{\omega} \leq L\right\}$ with $P(Q)>0$, where $L_{\omega}$ is a random variable constructed explicitly in the proof (see Remark 6.5), so that the condition (2.5) will be satisfied. Thus, by Theorem 2.1, if (2.6) and (2.7) are satisfied, then (2.8) and (2.9) hold 
true together with the characterization (2.10) of the case $\sigma=0$. The corresponding LIL follows, as well.

If I set $\mathcal{T}_{l}^{\omega}=\mathcal{F}_{l, \infty}^{\omega}$, then it follows from Section 6 that the conditions of Theorem 2.3 are also satisfied for random subshifts of finite type as above, and so Theorem 2.5 can be derived from Theorem 2.3, as well, though for a more restricted class of functions similar to the one in Theorem 2.6 below.

Finally, I shall consider expanding in average smooth random maps studied in $[\mathrm{KK}]$. In this set-up the $\Xi^{\omega}$ 's for all $\omega$ coincide with one compact connected $d$-dimensional $C^{2}$ Riemannian manifold $M$ (though a more general case of manifolds $M^{\omega}$ depending on $\omega$ may be considered, as well) and all $f_{\omega}: M \longrightarrow M$ are $C^{2}$ endomorphisms of $M$ such that

$$
\log \left\|D f_{\omega}^{-1}\right\|, \log \left\|D f_{\omega}\right\| \in L^{1}(\Omega, P),
$$

and

$$
\alpha=E_{P} \log \left\|D f_{\omega}^{-1}\right\|<0
$$

where $D f$ is the differential of $f$ and $\|\cdot\|$ is the supremum norm. Again, I define a random RPF operator $\mathcal{L}_{g}^{\omega}$ by $(2.24)$, and if the $g_{\omega}$ 's are Hölder continuous, i.e.

$$
\left|g_{\omega}(x)-g_{\omega}(y)\right| \leq K_{g}(\omega)(d(x, y))^{\kappa} \forall x, y \in M
$$

(where $d(\cdot, \cdot)$ is the distance function on $M$ ) for some $\kappa>0$ and a random variable $K_{g}(\omega)>0$ satisfying $(2.21)$, then (see $[K K]$ ) the random RPF theorem holds true, yielding a random variable $\lambda^{\omega}>0$, a function $h=h_{\omega}(x)>0$ on $M \times \Omega$, and probability measures $\nu^{\omega}, \mu^{\omega}$ on $M$ and $\mu$ on $M \times \Omega$ satisfying (2.25)-(2.27).

2.6. Theorem. Assume that the random transformation $f_{\omega}$ satisfies (2.28) and (2.29), the measures $\mu^{\omega}$ with properties (2.25)-(2.27) are constructed via the random RPF theorem from [KK] by a function $g$ satisfying (2.30), and a function $\varphi=\varphi_{\omega}(x)$ (for which the CLT is going to be proved) is Hölder continuous in $\xi$, i.e. it satisfies (2.30) with, say, the same exponent $\kappa>0$ and a random variable $K_{\varphi}(\omega)>0$ such that

$$
E_{P_{Q}}\left(\sum_{j=0}^{k_{1}(\omega)-1}\left(\left\|\varphi_{\theta^{j} \omega}\right\|+K_{\varphi}\left(\theta^{j} \omega\right)\right)\right)^{2}<\infty
$$

with $k_{1}$ given by (2.3). Then one can choose a set $Q$ in the form $Q=Q_{L}=\{\omega$ : $\left.L_{\omega} \leq L\right\}$ with $P(Q)>0$, where $L_{\omega}$ is a random variable constructed explicitly in the proof (see Remark 6.5), so that the conditions (i) and (ii) of Theorem 2.3 will be satisfied, and so (2.8) and (2.9) together with the characterization of the case $\sigma=0$ hold true, as well as the corresponding $L I L$.

If the closure of $\left\{f_{\omega}, \omega \in \Omega\right\}$ is a compact set of uniformly expanding endomorphisms, then one can construct a random Markov partition (see [BG1]) and reduce the problem to the case of random subshifts of finite type considered in Theorem 2.5. In the general case of random transformations expanding only in average (and in further generalizations) when symbolic representations are not available it is not clear how to construct the $\sigma$-algebras $\mathcal{F}_{m, n}^{\omega}$ and to exhibit the uniform mixing condition of Theorem 2.1, and one has to use Theorem 2.3 instead. If a version of the RPF theorem holds true so that an RPF operator (2.24) together with $h_{\omega}, \lambda_{\omega}, \nu^{\omega}$ satisfying (2.25) exist, then it is possible to write an explicit formula for the operator $u_{\omega}^{*}$ (see (6.29) in Section 6); but such a formula does not exist in more general 
situations, and then it is not clear how to verify the condition (ii) of Theorem 2.3, so in a number of cases Theorem 2.1 may be more readily applicable. Also the class of functions for which one can derive a CLT and LIL via Theorem 2.3 is usually more restrictive than what one gets via Theorem 2.1 .

Observe that Theorem 2.6 yields fiber-wise CLT and LIL for some deterministic skew product transformations. For instance, consider an expanding map of the 3-dimensional torus $\mathbb{T}^{3}=\mathbb{T}^{1} \times \mathbb{T}^{2}$ given by the formula $\tau(x, y)=\left(f_{y} x, \theta y\right)$, where $\theta$ is an ergodic automorphism of $\mathbb{T}^{2}$ and $f_{y} x=\gamma(y)+n(y) x(\bmod 1)$, where $\gamma(y) \in$ $\mathbb{R}, n(y) \in \mathbb{Z}_{+}$are measurable functions with $0<\int_{\mathbb{T}^{2}} \log n(y) d y<\infty$. Since both $\theta$ and the $f_{y}$ 's preserve the Lebesgue measures (denoted Leb below) on $\mathbb{T}^{2}$ and on $\mathbb{T}^{1}$, respectively, I can view the $f_{y}$ 's as "random" expanding maps of $\mathbb{T}^{1}$ with $\Omega=\mathbb{T}^{2}$, $P=$ Leb, $M=\mathbb{T}^{1}$, and $\mu^{y}=$ Leb (which is a "random" Gibbs measure corresponding to the function $\left.g_{y}=\log n(y)\right)$. Thus I arrive at a very particular case of the above situation, and Theorem 2.6 yields that for Leb-a.a.y, $\operatorname{Leb}\left\{x: \frac{1}{\sqrt{n}} \sum_{l=0}^{n-1} \psi \circ \tau^{l}(x, y)\right.$ $\leq a\}$ converges as $n \rightarrow \infty$ to the right hand side of (2.9), and the corresponding LIL follows, as well.

As another application of Theorem 2.6 I can write a CLT and an LIL for sums of digits in "random base" expansions (see [Ki4]).

2.7. Remark. An easy extension of the proofs of Theorems 2.1 and 2.3 yields a random functional CLT saying that for $P$-a.a. $\omega$ the process $\zeta_{t}^{(n)}=\frac{1}{\sqrt{n}} \sum_{i=0}^{[n t]} \psi \circ$ $\tau^{i}(\xi, \omega)$ on the space $\left(\Xi^{\omega}, \mu^{\omega}\right)$ weakly converges as $n \rightarrow \infty$ to the process $\sigma w_{t}$, where $w_{t}$ is the standard Wiener process (see Remark 2 on p. 510 in [Sh] and its proof on p. 517). Furthermore, combining methods of [PS] and the present paper, one can derive some invariance principle type results which also yield a $P$-a.s. law of the iterated logarithm for $\sum_{i=0}^{n} \psi \circ \tau^{i}(\xi, \omega)$ with respect to $\mu^{\omega}$. Note also that, using analyticity properties of the random RPF operators (see $[\mathrm{Gu}]$ ), one can derive similarly to $[\mathrm{Ki} 2]$ relativized (fiberwise) moderate deviations type results.

Important classes of continuous time dynamical systems can be modelled by suspension flows constructed by a well-mixing transformation and a sufficiently regular function. I introduce next the notion of random suspensions and formulate a corresponding CLT for them. The set-up consists of a measurable function $l=l_{\omega}(\xi)$ such that $C^{-1}(\omega)<l_{\omega}(\xi)<C(\omega)$ for some random variable $C(\omega)>0$ on $\Omega$, the space $Y=\left\{(s, \xi, \omega):(\xi, \omega) \in \Xi, 0 \leq s<l_{\omega}(\xi)\right\}$, and a continuous time (random) dynamical system (semi-flow) $\sigma^{t}$ defined by $\sigma^{t}(s, \xi, \omega)=(s+t, \xi, \omega)$ if $s+t<l_{\omega}(\xi)$ and $\sigma^{t}(s, \xi, \omega)=\left(u, \tau^{k}(\xi, \omega)\right)$ if $0 \leq u=s+t-\sum_{0 \leq i \leq k-1}\left(l \circ \tau^{i}\right)_{\omega}(\xi)$, where $k=k(s, t, \xi, \omega)=\max \left\{j \geq 1: s+t-\sum_{0 \leq i \leq j-1}\left(l \circ \tau^{i}\right)_{\omega}(\xi) \geq 0\right\}$. The semiflow $\sigma^{t}$ is called a suspension over $\tau$ with the ceiling function $l$. One can speak also of a family of random transformations $g_{\omega}^{t}$ defined by $g_{\omega}^{t}(s, \xi)=(s+t, \xi)$ if $\xi \in \Xi^{\omega}, s+t<l_{\omega}(\xi)$ and $g_{\omega}^{t}(s, \xi)=\left(u, f_{\omega}^{k} \xi\right)$ if $u$ and $k=k(s, t, \xi, \omega)$ are as above. Then $g_{\theta^{k} \omega}^{u} g_{\omega}^{t}(s, \xi)=g_{\omega}^{u+t}$.

Let $\mu$ be a $\tau$-invariant probability measure with the disintegration (1.1); then $\eta \in \mathcal{P}(Y)$ defined by $d \eta(s, \xi, \omega)=\frac{1}{l} d \mu^{\omega}(\xi) d P(\omega) d s$, where $\bar{l}=\int l_{\omega}(\xi) d \mu^{\omega}(\xi) d P(\omega)$, is $\sigma$-invariant. Let $\varphi=\varphi_{\omega}(\xi, s)$ be a measurable function on $Y, \psi=\psi_{\omega}=$ $\varphi_{\omega}-\int \varphi_{\omega} d \mu^{\omega}, \varphi_{\omega}^{(l)}(\xi)=\int_{0}^{l_{\omega}(\xi)} \varphi_{\omega}(\xi, s) d s$, and $\psi_{\omega}^{(l)}(\xi)=\varphi_{\omega}^{(l)}(\xi)-\int \varphi_{\omega}^{(l)} d \mu^{\omega}$. Let $\sigma$-algebras $\mathcal{F}_{m, n}^{\omega}$, a set $Q$, and hitting times $k_{i}$ be as in Theorem 2.1, and set $\Psi_{\omega}^{(l)}=\sum_{i=0}^{k_{1}(\omega)-1}\left(\psi^{(l)} \circ \tau^{i}\right)_{\omega}(\xi)$ and $R_{\omega}^{(l)}=\sum_{i=0}^{k_{1}(\omega)-1}\left|\psi^{(l)} \circ \tau^{i}(\xi, \omega)\right|$. 
2.8. Theorem. Suppose that the conditions of Theorem 2.1 are satisfied both for the function $\varphi^{(l)}$ and for the function $l$ in place of $\varphi$ there, and, in addition, $\sup _{\omega} C(\omega)<\infty$,

$$
\begin{gathered}
E_{\mu_{Q}}\left(R^{(l)}\right)^{8}<\infty \quad \text { and } E_{P_{Q}} \int_{0}^{l_{\omega}(\xi)}\left|\psi_{\omega}(\xi, s)\right| d s<\infty \\
\sum_{j=1}^{\infty} j^{4} \phi_{j}^{1 / 8}<\infty
\end{gathered}
$$

and

$$
\sum_{j=1}^{\infty} j^{4} \beta_{j}^{(8)}<\infty
$$

where $\beta_{j}^{(8)}=\left(E_{P_{Q}}\left(D_{k_{j}}^{(8)}\right)^{8}\right)^{1 / 8}$ and $D_{n}^{(8)}(\omega)=\left(E_{\mu^{\omega}}\left(\Psi_{\omega}^{(l)}-E_{\mu^{\omega}}\left(\Psi_{\omega}^{(l)} \mid \mathcal{F}_{0, n}^{\omega}\right)\right)^{8}\right)^{1 / 8}$. Then, $P$-a.s. for any number $a$,

$$
\lim _{t \rightarrow \infty} \mu^{\omega}\left\{\xi \in \Xi^{\omega}: \frac{1}{\sqrt{t}} \int_{0}^{t}\left(\psi \circ \sigma^{s}\right)_{\omega}(0, \xi) d s \leq a\right\}=\frac{1}{\sigma \sqrt{2 \pi}} \int_{-\infty}^{a} e^{-\frac{x^{2}}{2 \sigma^{2}}} d x
$$

where, P-a.s.,

$$
\begin{aligned}
& \sigma^{2}=\lim _{t \rightarrow \infty} \frac{1}{t} E_{\mu^{\omega}}\left(\int_{0}^{t}\left(\psi \circ \sigma^{s}\right)_{\omega} d s\right)^{2} \\
& =\bar{l}^{-1} P(Q)\left(E_{\mu_{Q}}\left(\Psi^{(l)}\right)^{2}+\sum_{l=1}^{\infty} E_{\mu_{Q}}\left(\Psi^{(l)}\left(\Psi^{(l)} \circ T^{j}\right)\right)\right) .
\end{aligned}
$$

If $\sigma>0$, then, combining methods of [DP] and the present paper, a corresponding LIL follows, as well.

\section{LIMIT THEOREMS UNDER MIXING CONDITIONS}

Set $N_{n}(\omega)=\max \left\{l: k_{l}(\omega)<n\right\}$; then

$$
\left|\sum_{l=0}^{n-1} \psi \circ \tau^{l}(\xi, \omega)-\sum_{j=0}^{N_{n}(\omega)-1} \Psi \circ T^{j}(\xi, \omega)\right| \leq R \circ T^{N_{n}(\omega)}(\xi, \omega),
$$

where $R_{\omega}(\xi)=R(\xi, \omega)=\sum_{j=0}^{k_{1}(\omega)-1}\left|\psi \circ \tau^{j}(\xi, \omega)\right|$. Next,

$$
\left(E_{\mu^{\omega}} R_{\omega}^{2}\right)^{1 / 2} \leq \sum_{j=0}^{k_{1}(\omega)-1} c\left(\theta^{j} \omega\right) \stackrel{\text { def }}{=} I(\omega)
$$

By (2.7) and the ergodic theorem, $P$-a.s.,

$$
\lim _{n \rightarrow \infty} \frac{1}{n} N_{n}(\omega)=P(Q) \text { and } \quad \lim _{n \rightarrow \infty} \frac{1}{\sqrt{n}} I\left(\Theta^{N_{n}(\omega)} \omega\right)=0 .
$$

Hence by (3.1) and (3.2), P-a.s.,

$$
\lim _{n \rightarrow \infty} \frac{1}{n} E_{\mu^{\omega}}\left(\sum_{j=0}^{n-1}\left(\psi \circ \tau^{j}\right)_{\omega}\right)^{2}=\lim _{n \rightarrow \infty} \frac{1}{n} E_{\mu^{\omega}}\left(\sum_{j=0}^{N_{n}(\omega)-1}\left(\Psi \circ T^{j}\right)_{\omega}\right)^{2},
$$


and for each $\varepsilon>0$ and $P$-a.a.w,

$$
\lim _{n \rightarrow \infty} \mu^{\omega}\left\{\xi: \frac{1}{\sqrt{n}}\left|\sum_{l=0}^{n-1} \psi \circ \tau^{l}(\xi, \omega)-\sum_{j=0}^{N_{n}(\omega)-1} \Psi \circ T^{j}(\xi, \omega)\right|>\varepsilon\right\}=0 .
$$

Thus it remains to deal with the asymptotical behavior of $\frac{1}{\sqrt{n}} \sum_{j=0}^{N_{n}(\omega)-1} \Psi \circ T^{j}(\xi, \omega)$, which is the same as of $\frac{1}{\sqrt{n}} \sum_{j=1}^{N_{n}(\omega)-1} \Psi \circ T^{j}(\xi, \omega)$, and so one has to prove results only for $P_{Q}-$ a.s. $\omega$. First, I shall study the variance

$$
\begin{aligned}
& E_{\mu^{\omega}}\left(\sum_{i=0}^{N_{n}(\omega)-1}\left(\Psi \circ T^{i}\right)_{\omega}\right)^{2}=\sum_{i, j=0}^{N_{n}(\omega)-1} E_{\mu^{\omega}}\left(\left(\Psi \circ T^{i}\right)_{\omega}\left(\Psi \circ T^{j}\right)_{\omega}\right) \\
& =\sum_{i=0}^{N_{n}(\omega)-1} E_{\mu^{\Theta^{i} \omega}} \Psi_{\Theta^{i} \omega}^{2}+2 \sum_{i=0}^{N_{n}(\omega)-2} \Phi_{N_{n}(\omega)-i}\left(\Theta^{i} \omega\right),
\end{aligned}
$$

where $\Phi_{k}(\omega)=\sum_{l=1}^{k-1} E_{\mu^{\omega}}\left(\Psi_{\omega}\left(\Psi \circ T^{l}\right)_{\omega}\right)$, and I use the equality $E_{\mu^{\omega}}(g \circ T)_{\omega}=$ $E_{\mu}{ }^{\Theta \omega} g_{\Theta \omega}$, which follows since $F_{\omega} \mu^{\omega}=\mu^{\Theta \omega}$. By Lemma 1 from Section 20 in [Bi1], whenever the function $V$ is $\mathcal{F}_{0, k_{i}(\omega)}^{\omega}$-measurable and the function $W$ is $\mathcal{F}_{k_{i+j}(\omega), \infty}^{\omega}$-measurable, for any $r, s>1, \frac{1}{r}+\frac{1}{s}=1$, one has

$$
\left|E_{\mu^{\omega}}(V W)-E_{\mu^{\omega}} V E_{\mu^{\omega}} W\right| \leq 2 \phi_{j}^{1 / r}\left(E_{\mu^{\omega}}|V|^{r}\right)^{1 / r}\left(E_{\mu^{\omega}}|W|^{s}\right)^{1 / s} .
$$

Since $\left(\Psi \circ T^{l}\right)_{\omega}$ is $\mathcal{F}_{k_{l}(\omega), \infty}^{\omega}$-measurable, then by (3.7) and the Cauchy-Schwarz inequality

$$
\begin{aligned}
& \left|E_{\mu^{\omega}}\left(\Psi_{\omega}\left(\Psi \circ T^{l}\right)_{\omega}\right)\right| \leq\left|E_{\mu^{\omega}}\left(\left(\Psi_{\omega}-E_{\mu^{\omega}}\left(\Psi_{\omega} \mid \mathcal{F}_{0, k_{[l / 2]}(\omega)}^{\omega}\right)\right)\left(\Psi \circ T^{l}\right)_{\omega}\right)\right| \\
& +\left|E_{\mu^{\omega}}\left(E_{\mu^{\omega}}\left(\Psi_{\omega} \mid \mathcal{F}_{0, k_{[l / 2]}(\omega)}^{\omega}\right)\left(\Psi \circ T^{l}\right)_{\omega}\right)\right| \\
& \leq\left(E_{\mu^{\omega}}\left(\Psi_{\omega}-E_{\mu^{\omega}}\left(\Psi_{\omega} \mid \mathcal{F}_{0, k_{[l / 2]}(\omega)}^{\omega}\right)\right)^{2}\right)^{1 / 2}\left(E_{\mu^{\omega}}\left(\Psi \circ T^{l}\right)_{\omega}^{2}\right)^{1 / 2} \\
& +2 \phi_{[l / 2]}^{1 / 2}\left(E_{\mu^{\omega}}\left(E_{\mu^{\omega}}\left(\Psi_{\omega} \mid \mathcal{F}_{0, k_{[l / 2]}(\omega)}^{\omega}\right)\right)^{2}\right)^{1 / 2}\left(E_{\mu^{\omega}}\left(\Psi \circ T^{l}\right)_{\omega}^{2}\right)^{1 / 2} \\
& \leq D_{k_{[l / 2]}(\omega)}(\omega) C\left(\Theta^{l} \omega\right)+2 \phi_{[l / 2]}^{1 / 2} C(\omega) C\left(\Theta^{l} \omega\right),
\end{aligned}
$$

where $[a]$ denotes the integral part of $a$. This together with the Cauchy-Schwarz inequality gives

$$
E_{P_{Q}}\left|E_{\mu^{\omega}}\left(\Psi_{\omega}\left(\Psi \circ T^{l}\right)_{\omega}\right)\right| \leq \beta_{[l / 2]}\left(E_{P_{Q}} C^{2}\right)^{1 / 2}+2 \phi_{[l / 2]}^{1 / 2} E_{P_{Q}} C^{2} .
$$

It follows from (2.4)-(2.7) and (3.9) that the series

$$
\Phi(\omega)=\sum_{l=1}^{\infty} E_{\mu^{\omega}}\left(\Psi_{\omega}\left(\Psi \circ T^{l}\right)_{\omega}\right)
$$


converges absolutely $P_{Q}-$ a.s. and in $L^{1}\left(Q, P_{Q}\right)$. Next, I claim that, $P_{Q}-$ a.s. and in $L^{1}\left(Q, P_{Q}\right)$,

$$
\lim _{m \rightarrow \infty} \frac{1}{m} \sum_{i=0}^{m-2} \Phi_{m-i}\left(\Theta^{i} \omega\right)=E_{P_{Q}} \Phi,
$$

which by the ergodic theorem is the same as

$$
\lim _{m \rightarrow \infty} \frac{1}{m} \sum_{i=0}^{m-2}\left(\Phi\left(\Theta^{i} \omega\right)-\Phi_{m-i}\left(\Theta^{i} \omega\right)\right)=0 .
$$

Indeed,

$$
\left|\Phi\left(\Theta^{i} \omega\right)-\Phi_{m-i}\left(\Theta^{i} \omega\right)\right| \leq \delta_{m-i}\left(\Theta^{i} \omega\right)
$$

where

$$
\delta_{j}(\omega)=\sum_{l=j}^{\infty}\left(D_{k_{[l / 2]}(\omega)}(\omega) C\left(\Theta^{l} \omega\right)+2 \phi_{[l / 2]}^{1 / 2} C(\omega) C\left(\Theta^{l} \omega\right)\right) .
$$

Since $\delta_{j}$ is monotone decreasing in $j$, then

$$
\sum_{i=0}^{m-2} \delta_{m-i}\left(\Theta^{i} \omega\right) \leq \sum_{i=0}^{m-j} \delta_{j}\left(\Theta^{i} \omega\right)+\sum_{i=m-j+1}^{m-2} \delta_{2}\left(\Theta^{i} \omega\right)
$$

By $(2.5)-(2.7), \delta_{2} \in L^{1}\left(Q, P_{Q}\right)$, and so $\lim _{m \rightarrow \infty} \frac{1}{m} \delta_{2}\left(\Theta^{m-i} \omega\right)=0 P-$ a.s. and in $L^{1}\left(Q, P_{Q}\right)$. Thus (3.14) together with the ergodic theorem yield that, $P$-a.s. and in $L^{1}\left(Q, P_{Q}\right)$,

$$
\limsup _{m \rightarrow \infty} \frac{1}{m} \sum_{i=0}^{m-2} \delta_{m-i}\left(\Theta^{i} \omega\right) \leq \lim _{m \rightarrow \infty} \frac{1}{m} \sum_{i=0}^{m-j} \delta_{j}\left(\Theta^{i} \omega\right)=E_{P_{Q}} \delta_{j} .
$$

Since $\delta_{j} \rightarrow 0$ as $j \rightarrow \infty$ both $P_{Q}-$ a.s. and in $L^{1}\left(Q, P_{Q}\right)$ and the left hand side of (3.15) does not depend on $j$, I derive that the left hand side of (3.15) is zero, which by (3.13) gives (3.12), and so (3.11) holds true. Finally, (3.1)-(3.4), (3.6), (3.11), and the ergodic theorem yield both (2.8) and

$$
\lim _{m \rightarrow \infty} \frac{1}{m} E_{\mu^{\omega}}\left(\sum_{i=0}^{m-1}\left(\Psi \circ T^{i}\right)_{\omega}\right)^{2}=E_{\mu_{Q}} \Psi^{2}+E_{P_{Q}} \Phi=\sigma^{2} / P(Q) .
$$

Next, I shall show that, $P_{Q}-$ a.s. for any number $a$,

$$
\lim _{m \rightarrow \infty} \mu^{\omega}\left\{\xi \in \Xi^{\omega}: \frac{1}{\sqrt{m}} \sum_{i=0}^{m-1} \Psi \circ T^{i}(\xi, \omega) \leq a\right\}=\frac{\sqrt{P(Q)}}{\sigma \sqrt{2 \pi}} \int_{-\infty}^{a} e^{-\frac{P(Q) x^{2}}{2 \sigma^{2}}} d x
$$

with $\sigma=0$ if and only if there exists a function $\eta$ on $\Xi_{Q}$ from $L^{2}\left(\Xi_{Q}, \mu_{Q}\right)$ such that $\mu_{Q}-$ a.s. (2.10) holds true. Actually, this last statement (i.e. the last assertion of Theorem 2.1) follows already from (3.16) (see $\S \S 2-5$ from Ch.18 in [IL] ). If $\sigma=0$ then (2.8) says that $\frac{1}{\sqrt{m}} \sum_{j=0}^{n-1} \psi_{\theta^{j} \omega} \circ f_{\omega}^{j}$ converges in $L^{2}\left(\Xi^{\omega}, \mu^{\omega}\right)$ (and so in $\mu^{\omega}$-measure) to zero, which yields the assertion of Theorem 2.1 for this case, so in what follows I assume that $\sigma^{2}>0$. Observe also that it suffices to prove (3.17) for each $a P$-a.s. and not $P$-a.s. simultaneously for all $a$, since the latter follows from the former in view of the monotonicity in $a$ of the left hand side of (3.17) and continuity in $a$ of the right hand side of (3.17), and so everything is determined already by rational $a$ 's. 
Set $\Psi_{\omega}^{(N)}=E_{\mu^{\omega}}\left(\Psi_{\omega} \mid \mathcal{F}_{0, k_{N}(\omega)}^{\omega}\right)$. I want to show first that if $\delta^{(N)}=\Psi-\Psi^{(N)}$, then, $P-$ a.s. for any $\varepsilon>0$,

$$
\lim _{N \rightarrow \infty} \limsup _{m \rightarrow \infty} \mu^{\omega}\left\{\xi:\left|\frac{1}{\sqrt{m}} \sum_{j=0}^{m-1} \delta^{(N)} \circ T^{j}(\xi, \omega)\right| \geq \varepsilon\right\}=0 .
$$

To do this I estimate

$$
E_{\mu^{\omega}}\left(\sum_{j=0}^{m-1}\left(\delta^{(N)} \circ T^{j}\right)_{\omega}\right)^{2}=\sum_{j=0}^{m-1} E_{\mu^{\Theta^{j} \omega}}\left(\delta_{\Theta^{j} \omega}^{(N)}\right)^{2}+2 \sum_{i=0}^{m-2} H_{m-i}^{(N)}\left(\Theta^{i} \omega\right),
$$

where $H_{k}^{(N)}=\sum_{l=1}^{k-1} E_{\mu^{\omega}}\left(\delta_{\omega}^{(N)}\left(\delta^{(N)} \circ T^{l}\right)_{\omega}\right)$. Taking into account that

$$
\begin{aligned}
& \left(E_{\mu^{\omega}}\left(\delta_{\omega}^{(N)}-E_{\mu^{\omega}}\left(\delta_{\omega}^{(N)} \mid \mathcal{F}_{0, k_{[l / 2]}(\omega)}^{\omega}\right)\right)^{2}\right)^{1 / 2} \\
& \leq\left(E_{\mu^{\omega}}\left(\Psi_{\omega}-E_{\mu^{\omega}}\left(\Psi_{\omega} \mid \mathcal{F}_{0, k_{[l / 2]}(\omega)}^{\omega}\right)\right)^{2}\right)^{1 / 2} \\
& +\left(E_{\mu^{\omega}}\left(\Psi_{\omega}^{(N)}-E_{\mu^{\omega}}\left(\Psi_{\omega}^{(N)} \mid \mathcal{F}_{0, k_{[l / 2]}(\omega)}^{\omega}\right)\right)^{2}\right)^{1 / 2} \leq 2 D_{k_{[l / 2]}(\omega)}(\omega),
\end{aligned}
$$

I derive in the same way as in (3.8) that

$$
\begin{aligned}
& \left|E_{\mu^{\omega}}\left(\delta_{\omega}^{(N)}\left(\delta^{(N)} \circ T^{l}\right)_{\omega}\right)\right| \leq\left(E_{\mu^{\omega}}\left(\delta_{\omega}^{(N)}-E_{\mu^{\omega}}\left(\delta_{\omega}^{(N)} \mid \mathcal{F}_{0, k_{[l / 2]}(\omega)}^{\omega}\right)\right)^{2}\right)^{1 / 2} \\
& \times\left(E_{\mu_{\omega}}\left(\delta^{(N)} \circ T^{l}\right)_{\omega}^{2}\right)^{1 / 2}+2 \phi_{[l / 2]}^{1 / 2}\left(E_{\mu^{\omega}}\left(\delta_{\omega}^{(N)}\right)^{2}\right)^{1 / 2}\left(E_{\mu_{\omega}}\left(\delta^{(N)} \circ T^{l}\right)_{\omega}^{2}\right)^{1 / 2} \\
& \leq 2 D_{k_{[l / 2]}(\omega)}(\omega) D_{k_{N}(\omega)}\left(\Theta^{l} \omega\right)+2 \phi_{[l / 2]}^{1 / 2} D_{k_{N}(\omega)}(\omega) D_{k_{N}(\omega)}\left(\Theta^{l} \omega\right) .
\end{aligned}
$$

This together with the Cauchy-Schwarz inequality gives

$$
E_{P_{Q}}\left|E_{\mu^{\omega}}\left(\delta_{\omega}^{(N)}\left(\delta^{(N)} \circ T^{l}\right)_{\omega}\right)\right| \leq 2 \beta_{[l / 2]} \beta_{N}+2 \phi_{[l / 2]}^{1 / 2} \beta_{N}^{2} .
$$

It follows from (2.5) and (2.6) that the series

$$
H^{(N)}(\omega)=\sum_{l=1}^{\infty} E_{\mu^{\omega}}\left(\delta_{\omega}^{(N)}\left(\delta^{(N)} \circ T^{l}\right)_{\omega}\right)
$$

converges $P$-a.s. and in $L^{1}\left(Q, P_{Q}\right)$, and so I derive similarly to (3.16) that, $P$-a.s. and in $L^{1}\left(Q, P_{Q}\right)$,

$$
\lim _{m \rightarrow \infty} \frac{1}{m} E_{\mu^{\omega}} \sum_{j=0}^{m-1}\left(\delta^{(N)} \circ T_{\omega}^{j}\right)^{2}=\beta_{N}^{2}+E_{P_{Q}} H^{(N)} .
$$

Now (3.18) follows from (3.21)-(3.23) together with the Chebyshev inequality. Similarly to above I derive also that, $P$-a.s. and in $L^{1}\left(Q, P_{Q}\right)$,

$$
\lim _{m \rightarrow \infty} \frac{1}{m} E_{\mu^{\omega}}\left(\sum_{j=0}^{m-1}\left(\Psi^{(N)} \circ T^{j}\right)_{\omega}\right)^{2}=E_{\mu_{Q}}\left(\Psi^{(N)}\right)^{2}+E_{P_{Q}} \Phi^{(N)} \stackrel{\text { def }}{=}\left(\sigma^{(N)}\right)^{2},
$$


where $\Phi^{(N)}(\omega)=\sum_{l=1}^{\infty} E_{\mu^{\omega}}\left(\Psi_{\omega}^{(N)}\left(\Psi^{(N)} \circ T^{l}\right)_{\omega}\right)$, and it follows from (2.5), (2.6), and (3.22) that

$$
\lim _{N \rightarrow \infty} \lim _{m \rightarrow \infty} \frac{1}{m} E_{\mu^{\omega}}\left(\sum_{j=0}^{m-1}\left(\Psi^{(N)} \circ T^{j}\right)_{\omega}\right)^{2}=\lim _{N \rightarrow \infty}\left(\sigma^{(N)}\right)^{2}=\frac{\sigma^{2}}{P(Q)} .
$$

Since I am assuming that $\sigma^{2}>0$, then for all $N$ large enough $\left(\sigma^{(N)}\right)^{2}>0$; and only such $N$ will be considered in what follows. Therefore, in order to obtain (3.17) it remains to show that, $P_{Q}-$ a.s. for each number $a$ and any $N$ large enough,

$$
\lim _{m \rightarrow \infty} \mu^{\omega}\left\{\xi \in \Xi^{\omega}: \frac{1}{\sqrt{m}} \sum_{j=0}^{m-1} \Psi^{(N)} \circ T^{j}(\xi, \omega) \leq a\right\}=\frac{\sqrt{P(Q)}}{\sigma^{(N)} \sqrt{2 \pi}} \int_{-\infty}^{a} e^{-\frac{P(Q) x^{2}}{2\left(\sigma^{(N)}\right)^{2}}} d x .
$$

Set $Z^{(n)}=Z_{\omega}^{(n)}=\sum_{j=0}^{n-1}\left(\Psi^{(N)} \circ T^{j}\right)_{\omega}, Z^{(m, n)}=Z_{\omega}^{(m, n)}=Z_{\omega}^{(n)}-Z_{\omega}^{(m)}$ for $n \geq m$, and $\chi^{(m, n)}(u)=\chi_{\omega}^{(m, n)}(u)=E_{\mu \omega} e^{i u Z_{\omega}^{(m, n)}}$, where $i=\sqrt{-1}$. Observe that

$$
\left(\Psi^{(N)} \circ T^{j}\right)_{\omega}=E_{\mu^{\Theta^{j} \omega}}\left(\Psi_{\Theta^{j} \omega} \mid \mathcal{F}_{0, k_{N}\left(\Theta^{j} \omega\right)}^{\Theta^{j} \omega}\right) \circ F_{\omega}^{j}=E_{\mu^{\omega}}\left(\Psi \circ T^{j} \mid \mathcal{F}_{k_{j}(\omega), k_{j+N}(\omega)}^{\omega}\right),
$$

and so $Z_{\omega}^{(m, n)}$ is $\mathcal{F}_{k_{m}(\omega), k_{n+N-1}(\omega)}^{\omega}$-measurable. Set

$$
\begin{aligned}
& A_{j}(\omega)=E_{\mu^{\omega}}\left(\left(e^{i u Z_{\omega}^{((k-1) l, k l)}}-1\right)\left(e^{i u\left(\Psi^{(N)} \circ T^{k l+j}\right)_{\omega}}-1\right) e^{i u Z_{\omega}^{(k l+j+1, m l)}}\right) \\
& -E_{\mu^{\omega}}\left(e^{i u Z_{\omega}^{((k-1) l, k l)}}-1\right) E_{\mu^{\omega}}\left(\left(e^{i u\left(\Psi^{(N)} \circ T^{k l+j}\right)_{\omega}}-1\right) e^{i u Z_{\omega}^{(k l+j+1, m l)}}\right) .
\end{aligned}
$$

Then the Cauchy-Schwarz inequality and $\left|e^{i u x}-1\right| \leq|u x|$ yield that for any $j=$ $0,1, \ldots$,

$$
A_{j}(\omega) \leq 2 u^{2}\left(E_{\mu^{\omega}}\left(Z_{\omega}^{((k-1) l, k l)}\right)^{2}\right)^{1 / 2} C\left(\Theta^{k l+j} \omega\right)
$$

and using, in addition, (3.7), I derive for $j=N, N+1, \ldots$ that

$$
A_{j}(\omega) \leq 2 u^{2} \phi_{j-N+1}^{1 / 2}\left(E_{\mu^{\omega}}\left(Z_{\omega}^{((k-1) l, k l)}\right)^{2}\right)^{1 / 2} C\left(\Theta^{k l+j} \omega\right) .
$$

Thus, similarly to Lemma 3 from [Co], it follows that, for any $k=1,2, \ldots, m-1$ and $J=(m-k) l$,

$$
\begin{aligned}
& \left|\chi_{\omega}^{((k-1) l, m l)}(u)-\chi_{\omega}^{((k-1) l, k l)}(u) \chi_{\omega}^{(k l, m l)}(u)\right| \leq\left|\sum_{j=0}^{J-1} A_{j}(\omega)\right| \\
& \leq 2 u^{2}\left(E_{\mu^{\omega}}\left(Z_{\omega}^{((k-1) l, k l)}\right)^{2}\right)^{1 / 2}\left(\sum_{j=0}^{N-1} C\left(\Theta^{k l+j} \omega\right)+\sum_{j=N}^{J-1} \phi_{j-N+1}^{1 / 2} C\left(\Theta^{k l+j} \omega\right)\right) .
\end{aligned}
$$


Therefore by the Cauchy-Schwarz inequality

$$
\begin{aligned}
&\left|\chi_{\omega}^{(0, m l)}(u)-\prod_{k=1}^{m} \chi_{\omega}^{((k-1) l, k l)}(u)\right| \\
& \leq\left|\sum_{k=1}^{m-1}\left(\chi_{\omega}^{((k-1) l, m l)}(u)-\chi_{\omega}^{((k-1) l, k l)}(u) \chi_{\omega}^{(k l, m l)}(u)\right) \prod_{j=1}^{k-1} \chi_{((j-1) l, j l)}(u)\right| \\
& \leq 2 u^{2}\left(\sum_{k=1}^{m-1}\left(E_{\mu}\left(Z_{\omega}^{((k-1) l, k l)}\right)^{2}\right)^{1 / 2}\right. \\
& \times\left(\sum_{k=1}^{m}\left(\sum_{j=0}^{N} C\left(\Theta^{k l+j} \omega\right)+\sum_{j=N}^{\infty} \phi_{j-N+1}^{1 / 2}\left(C\left(\Theta^{k l+j} \omega\right)\right)^{2}\right)^{1 / 2}\right. \\
& \leq 2 u^{2}\left(\sum_{k=1}^{m-1}\left(E_{\mu^{\omega}}\left(Z_{\omega}^{((k-1) l, k l)}\right)^{2}\right)^{1 / 2}\left(\sum_{j=1}^{\infty} \phi_{j}^{1 / 2}\right)^{1 / 2}\left(\sum_{k=1}^{m} G\left(\Theta^{k l+N-1} \omega\right)\right)^{1 / 2},\right.
\end{aligned}
$$

where $G(\omega)=\sum_{j=1}^{\infty} \phi_{j}^{1 / 2} C^{2}\left(\Theta^{j} \omega\right)$. Since $\Theta$ preserves $P_{Q}$, then by (2.4), (2.5), (2.7), and the monotone convergence theorem I obtain that

$$
E_{P_{Q}} G=E_{P_{Q}} C^{2} \sum_{j=1}^{\infty} \phi_{j}^{1 / 2}<\infty
$$

By the ergodic theorem

$$
\lim _{m \rightarrow \infty} \frac{1}{m} \sum_{k=1}^{m-1} G \circ \Theta^{k l+N-1}(\omega)=E_{P_{Q}}\left(G \circ \Theta^{N-1} \mid J_{l}\right),
$$

where $J_{l}$ is the $\sigma$-algebra of $\Theta^{l}$-invariant subsets of $Q$.

Now let $m=[n / l]$. Since

$$
\frac{1}{\sqrt{n}}\left(E_{\mu^{\omega}}\left(Z_{\omega}^{(n)}-Z_{\omega}^{(m l)}\right)^{2}\right)^{1 / 2} \leq \frac{\sum_{j=0}^{l} C\left(\Theta^{m l+j} \omega\right)}{\sqrt{m l}},
$$

then it follows by $(2.4)$ and (2.7) that, for $P_{Q}-$ a.a.w and any $\varepsilon>0$,

$$
\mu^{\omega}\left\{\xi: \frac{1}{\sqrt{n}}\left|Z_{\omega}^{(n)}(\xi)-Z_{\omega}^{(m l)}(\xi)\right|>\varepsilon\right\} \longrightarrow 0 \quad \text { as } \quad n \rightarrow \infty,
$$

while $l$ remains fixed. Next, applying (3.32) I obtain

$$
\begin{aligned}
& \left|\chi_{\omega}^{(0, m l)}\left(\frac{u}{\sqrt{n}}\right)-\prod_{k=1}^{m} \chi_{\omega}^{((k-1) l, k l)}\left(\frac{u}{\sqrt{n}}\right)\right| \leq \frac{2 u^{2}}{l}\left(\frac{1}{m} \sum_{k=1}^{m-1} E_{\mu^{\omega}}\left(Z_{\omega}^{((k-1) l, k l)}\right)^{2}\right)^{\frac{1}{2}} \\
& \times\left(\sqrt{N}\left(\frac{1}{m} \sum_{k=1}^{m-1} \sum_{j=0}^{N-1} C^{2}\left(\Theta^{k l+j} \omega\right)\right)^{\frac{1}{2}}+\left(\sum_{j=1}^{\infty} \phi_{j}^{\frac{1}{2}}\right)^{\frac{1}{2}}\left(\frac{1}{m} \sum_{k=1}^{m-1} G\left(\Theta^{k l+N-1} \omega\right)\right)^{\frac{1}{2}}\right) .
\end{aligned}
$$

Since for any Borel function $q$,

$$
E_{\mu^{\omega}} q\left(Z_{\omega}^{((k-1) l, k l)}\right)=E_{\mu^{\Theta(k-1) l_{\omega}}} q\left(Z_{\Theta^{(k-1) l} \omega}^{(l)}\right),
$$


then by the ergodic theorem as $m \rightarrow \infty$ the right hand side of (3.37) converges $P_{Q}-$ a.s. and in $L^{1}\left(Q, P_{Q}\right)$ to

$$
\begin{aligned}
& \frac{2 u^{2}}{\sqrt{l}}\left\{\frac{1}{l} E_{P_{Q}}\left(E_{\mu \omega}\left(Z_{\omega}^{(l)}\right)^{2} \mid J_{l}\right)\right\}^{1 / 2} \\
& \times\left\{\left(E_{P_{Q}}\left(N \sum_{j=0}^{N-1} C^{2} \circ \Theta^{j} \mid J_{l}\right)\right)^{1 / 2}+\left(\sum_{j=1}^{\infty} \phi_{j}^{\frac{1}{2}}\right)^{\frac{1}{2}}\left(E_{P_{Q}}\left(G \circ \Theta^{N-1} \mid J_{l}\right)\right)^{\frac{1}{2}}\right\} .
\end{aligned}
$$

By $(3.25)$, in $L^{1}\left(Q, P_{Q}\right)$,

$$
\lim _{l \rightarrow \infty} \frac{1}{l} E_{P_{Q}}\left(E_{\mu^{\omega}}\left(Z_{\omega}^{(l)}\right)^{2} \mid J_{l}\right)=\left(\sigma^{(N)}\right)^{2} .
$$

Choose $l=l_{\nu}=\nu$ ! for $\nu=1,2, \ldots$; then $J_{l_{\nu+1}} \supset J_{l_{\nu}}$, and so, by the martingale convergence theorem the term in the second braces in (3.39) converges $P_{Q}-$ a.s. as $l_{\nu} \rightarrow \infty$. By (3.40) the term in the first braces in (3.39) converges in $L^{1}\left(Q, P_{Q}\right)$, and so I can choose a subsequence $\nu_{j}$ so that along the subsequence $l_{\nu_{j}}$ this term converges $P_{Q}-$ a.s. In view of the coefficient $l^{-1 / 2}$ in front, the whole expression in (3.39) converges $P_{Q}$-a.s. to zero along $l=l_{\nu_{j}} \rightarrow \infty$, and so $P_{Q}-$ a.s.,

$$
\chi_{\omega}^{(0, n)}\left(\frac{u}{\sqrt{n}}\right)-\prod_{k=1}^{n} \chi_{\omega}^{\left((k-1) l_{\nu_{j}}, k l_{\nu_{j}}\right)}\left(\frac{u}{\sqrt{n}}\right) \longrightarrow 0
$$

as, first, $m \rightarrow \infty$ and, then, $l=l_{\nu_{j}} \rightarrow \infty$. Since $\chi_{\omega}^{(0, n)}\left(\frac{u}{\sqrt{n}}\right)$ does not depend directly on $m$ and $l_{\nu_{j}}$, while $m=m_{\nu_{j}}=\left[\frac{n}{l_{\nu_{j}}}\right]$, and the product in (3.41) is the characteristic function of a sum of (not identically distributed) independent random variables then in order to complete the proof of Theorem 2.1 I can use a CLT for arrays (see Theorem 7.1 in [Bi1], Theorem 27.2 in [Bi2], or $\$ 4$ of Ch.III in [Sh]) provided the Lindenberg condition saying that $P$-a.s. for any $\varepsilon>0$ (recall that I assume $\left(\sigma^{(N)}\right)^{2}>0$ in $\left.(3.25)\right)$,

$$
\lim _{l_{\nu_{j}} \rightarrow \infty} \lim _{m_{\nu_{j}} \rightarrow \infty} \frac{1}{m_{\nu_{j}} l_{\nu_{j}}} \sum_{k=1}^{m_{\nu_{j}}} \int_{\left\{\left|Z_{\omega}^{\left((k-1) l_{\nu_{j}}, k l_{\nu_{j}}\right)}\right|>\varepsilon \sqrt{m_{\nu_{j}} l_{\nu_{j}}}\right\}}\left(Z_{\omega}^{\left((k-1) l_{\nu_{j}}, k l_{\nu_{j}}\right)}\right)^{2} d \mu^{\omega}=0
$$

holds true. In order to obtain (3.42), observe that in view of (3.38) by the ergodic theorem for any $K>0, P_{Q}-$ a.s. and in $L^{1}\left(Q, P_{Q}\right)$,

$$
\begin{aligned}
& \lim _{m \rightarrow \infty} \frac{1}{m} \sum_{k=1}^{m} E_{\mu^{\omega}}\left(\left(Z_{\omega}^{((k-1) l, k l))}\right)^{2} \mathbb{I}_{\left|Z_{\omega}^{((k-1) l, k l))}\right|>K}\right) \\
& =E_{P_{Q}}\left(E_{\mu^{\omega}}\left(\left(Z_{\omega}^{(l)}\right)^{2} \mathbb{I}_{\left|Z_{\omega}^{(l)}\right|>K}\right) \mid J_{l}\right) .
\end{aligned}
$$

If $m_{\nu_{j}}$ is large enough then $\varepsilon \sqrt{m_{\nu_{j}} l_{\nu_{j}}}>K$, and so, $P_{Q}-$ a.s.,

$$
\begin{aligned}
& \limsup _{m_{\nu_{j}} \rightarrow \infty} \frac{1}{m_{\nu_{j}} l_{\nu_{j}}} \sum_{k=1}^{m_{\nu_{j}}} \int_{\left\{\left|Z_{\omega}^{\left((k-1) l_{\nu_{j}}, k l_{\nu_{j}}\right)}\right|>\varepsilon \sqrt{m_{\nu_{j}} l_{\nu_{j}}}\right\}}\left(Z_{\omega}^{\left((k-1) l_{\nu_{j}}, k l_{\nu_{j}}\right)}\right)^{2} d \mu^{\omega} \\
& \leq E_{P_{Q}}\left(E_{\mu}\left(Z^{\left(l_{\nu_{j}}\right)}\right)^{2} \mathbb{I}_{\left|Z^{\left(l_{j}\right)}\right|>K} \mid J_{l_{\nu_{j}}}\right)
\end{aligned}
$$


for any $K>0$. Since the left hand side of (3.44) does not depend on $K$ and the right hand side of (3.44) tends to zero as $K \rightarrow \infty$, I conclude that both the limit in (4.44) and the limit in (3.42) equal zero.

The invariance principle for the LIL part of Theorem 2.1 will be derived in the next section via a martingale differences representation by some modification of methods from $[\mathrm{HH}]$, which will provide another proof of the CLT part of Theorem 2.1 , as well.

In order to prove Proposition 2.2, observe that

$$
E_{P_{Q}}\left(\sum_{j=0}^{k_{1}-1} c \circ \theta^{j}\right)^{2} \leq c^{2} E_{P_{Q}} k_{1}^{2}=\frac{1}{P(Q)} \sum_{j=0}^{\infty} P\left\{k_{1}>j\right\}
$$

if $\sup _{\omega} c(\omega)=c<\infty$, and

$$
\begin{aligned}
& E_{P_{Q}}\left(\sum_{j=0}^{k_{1}-1} c \circ \theta^{j}\right)^{2} \leq \sum_{j=0}^{\infty}\left(E_{P_{Q}} \mathbb{I}_{k(\omega)>j} c^{2} \circ \theta^{j}\right)^{1 / 2} \\
& \leq\left(E_{P_{Q}} c^{4}\right)^{1 / 4}(P(Q))^{-1 / 4} \sum_{j=0}^{\infty}\left(P\left\{k_{1}(\omega)>j\right\}\right)^{1 / 4} .
\end{aligned}
$$

Employing Lemma 1.1 from [VR] (or applying repeatedly Lemma 2 from Section 27 of [Bi2]), I obtain

$$
\begin{aligned}
& P\left\{k_{1}>n\right\}=P\left\{\theta^{j} \notin Q, j=1,2, \ldots, n\right\} \leq E_{P} \prod_{\frac{1}{3} \sqrt{n} \geq l \geq 0} \mathbb{I}_{\Omega \backslash \theta^{-(1+3 l[\sqrt{n}])} Q} \\
& \leq E_{P} \prod_{\frac{1}{3} \sqrt{n} \geq l \geq 0} E_{P}\left(I_{\Omega \backslash \theta^{-(1+3 l[\sqrt{n}])} Q} \mid \mathcal{G}_{1+(3 l-1)[\sqrt{n}], 1+(3 l+1)[\sqrt{n}]}\right) \\
& +\sum_{\frac{1}{3} \sqrt{n} \geq l \geq 0} E_{P}\left|\mathbb{I}_{\theta^{-(1+3 l[\sqrt{n}])} Q}-E_{P}\left(I_{\theta^{-(1+3 l[\sqrt{n}])} Q} \mid \mathcal{G}_{1+(3 l-1)[\sqrt{n}], 1+(3 l+1)[\sqrt{n}]}\right)\right| \\
& \leq 6 \sqrt{n} \alpha_{([\sqrt{n}])}+(1-P(Q))^{\left[\frac{1}{3} \sqrt{n}\right]}+\sum_{\frac{1}{3} \sqrt{n} \geq l \geq 0} E_{P}\left|\mathbb{I}_{Q}-E_{P}\left(\mathbb{I}_{Q} \mid \mathcal{G}_{-[\sqrt{n}],[\sqrt{n}]}\right)\right| \\
& \leq 6 \sqrt{n}\left(\alpha_{([\sqrt{n}])}+\gamma_{([\sqrt{n}])}\right)+(1-P(Q))^{\left[\frac{1}{3} \sqrt{n}\right]}
\end{aligned}
$$

with $\alpha_{n}$ and $\gamma_{n}$ defined in (2.11) and (2.12). This together with (3.45) and (3.46) yields Proposition 2.2.

\section{Limit TheOREMS Via MARTINGALE DifFERENCES}

In order to obtain martingale difference representations in the framework of Theorem 2.1 I shall pass first to an invertible case by means of the "random natural extension construction". Namely, introduce the spaces

$$
\hat{\Xi}^{\omega}=\left\{\hat{\xi}: \hat{\xi}=\left(\ldots, \xi_{-1}, \xi_{0}, \xi_{1}, \ldots\right), \xi_{i} \in \Xi^{\theta^{i} \omega}, \xi_{i+1}=f_{\theta^{i} \omega} \xi_{i} \forall i \in \mathbb{Z}\right\},
$$

$\hat{\Xi}=\left\{(\hat{\xi}, \omega): \omega \in \Omega, \hat{\xi} \in \hat{\Xi}^{\omega}\right\}$, and $\hat{\Xi}_{Q}=\left\{(\hat{\xi}, \omega): \omega \in Q, \hat{\xi} \in \hat{\Xi}^{\omega}\right\}$. Then the twosided shift $\hat{f}_{\omega}: \hat{\Xi}^{\omega} \rightarrow \hat{\Xi}^{\theta \omega}$ defined by $\left(\hat{f}_{\omega} \hat{\xi}\right)_{i}=\xi_{i+1}$ is invertible and $f_{\omega} \iota_{\omega}=\iota_{\theta \omega} \hat{f}_{\omega}$ where, $\iota_{\omega}: \hat{\Xi}^{\omega} \rightarrow \Xi^{\omega}$ acting by $\iota_{\omega}(\hat{\xi})=\xi_{0}, \hat{\xi}=\left(\ldots, \xi_{-1}, \xi_{0}, \xi_{1}, \ldots\right)$ is the natural projection. Set $\hat{\mathcal{F}}_{m, n}^{\omega}=\iota_{\omega}^{-1} \mathcal{F}_{m, n}^{\omega}, n \geq m \geq 0$, and $\hat{\mathcal{F}}_{-k, n}^{\omega}=\hat{f}_{\theta^{-k} \omega}^{k} \hat{\mathcal{F}}_{0, n+k}^{\theta^{-k} \omega}$ for $k \geq 0$. 
Observe that $\hat{\mathcal{F}}_{m, n}^{\omega} \subset \hat{\mathcal{F}}_{m^{\prime}, n^{\prime}}^{\omega}$ if $m^{\prime} \leq m$, and $n^{\prime} \geq n$ and $\hat{f}_{\omega}^{-1} \hat{\mathcal{F}}_{m, n}^{\theta \omega}=\hat{\mathcal{F}}_{m+1, n+1}^{\omega}$, and so for all $k \geq 1$,

$$
\hat{\mathcal{F}}_{-(k-1), n}^{\omega}=\hat{f}_{\theta^{-k} \omega}^{k} \hat{f}_{\theta^{-k} \omega}^{-1} \hat{\mathcal{F}}_{0, n+k-1}^{\theta^{-(k-1)} \omega}=\hat{f}_{\theta^{-k} \omega}^{k} \hat{\mathcal{F}}_{1, n+k}^{\theta^{-k} \omega} \subset \hat{f}_{\theta^{-k} \omega}^{k} \hat{\mathcal{F}}_{0, n+k}^{\theta^{-k} \omega}=\hat{\mathcal{F}}_{-k, n}^{\omega} .
$$

By $\hat{\mathcal{F}}_{-\infty, n}^{\omega}$ I denote the minimal $\sigma$-algebra containing $\bigcup_{k \geq 0} \hat{\mathcal{F}}_{-k, n}^{\omega}$. Let $\mu$ be a $\tau$-invariant probability measure with the disintegration (1.1). For any $\Gamma \in \hat{\mathcal{F}}_{m, n}^{\omega}$ with $0 \leq m \leq n \leq \infty$ there exists $G \in \mathcal{F}_{m, n}^{\omega}$ such that $\Gamma=\iota_{\omega}^{-1} G$, and I set $\hat{\mu}^{\omega}(\Gamma)=\mu^{\omega}(G)$. If $\Gamma \in \hat{\mathcal{F}}_{m, n}^{\omega}$ with $m<0$ and $-\infty<m \leq n \leq \infty$, then there exists $G \in \mathcal{F}_{0, n-m}^{\theta^{m} \omega}$ such that $\Gamma=\left(\hat{f}_{\omega}^{m}\right)^{-1} \iota_{\theta^{m} \omega}^{-1} G$. For such $\Gamma$ I define $\hat{\mu}^{\omega}(\Gamma)=\mu^{\theta^{m} \omega}(G)$ and I extend $\hat{\mu}^{\omega}$ to the whole $\hat{\mathcal{F}}_{-\infty, \infty}^{\omega}$ by monotone limits. It follows that $\hat{f}_{\omega} \hat{\mu}^{\omega}=\hat{\mu}^{\theta \omega}$ and $\hat{f}_{\omega}^{-1} \hat{\mu}^{\theta \omega}=\hat{\mu}^{\omega}$. Set also $\hat{\varphi}_{\omega}=\varphi_{\omega} \circ \iota_{\omega}, \hat{\psi}_{\omega}=\psi_{\omega} \circ \iota_{\omega}, \hat{\Psi}_{\omega}=\Psi_{\omega} \circ \iota_{\omega}, \hat{\tau}(\hat{\xi}, \omega)=$ $\left(\hat{f}_{\omega} \hat{\xi}, \theta \omega\right), \hat{F}_{\omega}=\hat{f}_{\omega}^{k_{1}(\omega)}$ and $\hat{T}(\hat{\xi}, \omega)=\hat{\tau}^{k_{1}(\omega)}(\hat{\xi}, \omega)$.

Let $k_{i}(\omega)$ be as before for $i \geq 0$ and $k_{i}(\omega)=\max \left\{k<k_{i+1}(\omega): \theta^{k} \omega \in Q\right\}$ for $i<0$. Take arbitrary $A \in \hat{\mathcal{F}}_{k_{l}(\omega), k_{i}(\omega)}^{\omega}$ and $B \in \hat{\mathcal{F}}_{k_{i+j}(\omega), \infty}^{\omega}$ with $l<0$ and $l \leq i \leq j$; then there exist $G \in \mathcal{F}_{0, k_{i}(\omega)-k_{l}(\omega)}^{\theta^{k_{l}(\omega)}=\mathcal{F}_{0, k_{i-l}\left(\Theta^{l} \omega\right)}^{\Theta^{l} \omega}}$ and $H \in \mathcal{F}_{k_{i+j-l}\left(\Theta^{l} \omega\right), \infty}$ such that $A=\hat{F}_{\Theta^{l} \omega^{\iota} \Theta^{l} \omega}^{-l} G$ and $B=\hat{F}_{\Theta^{l} \omega^{l} \theta^{l} \omega}^{-l} H$. It follows that

$$
\left|\frac{\hat{\mu}^{\omega}(A \cap B)}{\hat{\mu}^{\omega}(A)}-\hat{\mu}^{\omega}(B)\right|=\left|\frac{\mu^{\Theta^{l} \omega}(G \cap H)}{\mu^{\Theta^{l} \omega}(G)}-\mu^{\Theta^{l} \omega}(H)\right| \leq \phi_{j} .
$$

Since (4.1) is preserved under monotone limits, I conclude that it remains true for any $A \in \hat{\mathcal{F}}_{-\infty, k_{i}(\omega)}^{\omega}$. Set

$$
I_{i}^{(l)}(\omega)=E_{\hat{\mu}^{\omega}}\left(\hat{\Psi} \circ \hat{T}^{l} \mid \hat{\mathcal{F}}_{-\infty, k_{-i}(\omega)}^{\omega}\right)
$$

and

$$
J_{i}^{(l)}(\omega)=\hat{\Psi} \circ \hat{T}^{l}-E_{\hat{\mu}^{\omega}}\left(\hat{\Psi} \circ \hat{T}^{l} \mid \hat{\mathcal{F}}_{-\infty, k_{-i}(\omega)}^{\omega}\right),
$$

where $\hat{T}^{l}(\hat{\xi}, \omega)=\left(\left(\hat{F}_{\Theta^{l} \omega}^{-l}\right)^{-1} \hat{\xi}, \Theta^{l} \omega\right)$ for $l<0$, and define

$$
\begin{aligned}
Y_{\omega} & =\sum_{l=0}^{\infty}\left(I_{0}^{(l)}(\omega)-I_{1}^{(l)}\right)+\sum_{l=-\infty}^{-1}\left(J_{1}^{(l)}(\omega)-J_{0}^{(l)}(\omega)\right), \\
g_{\omega} & =\sum_{l=0}^{\infty} I_{1}^{(l)}(\omega)-\sum_{l=-\infty}^{-1} J_{1}^{(l)}(\omega) .
\end{aligned}
$$

I claim that for $P_{Q}-$ a.a. $\omega$ all series above converge in $L^{2}\left(\hat{\Xi}^{\omega}, \hat{\mu}^{\omega}\right)$. Indeed, for $l \geq 0$,

$$
I_{0}^{(l)}(\omega)=E_{\hat{\mu}^{\Theta l} \omega}\left(\hat{\Psi}_{\Theta^{l} \omega} \mid \hat{\mathcal{F}}_{-\infty, \Theta_{-l}\left(\Theta^{l} \omega\right)}^{\Theta^{l} \omega}\right) \circ \hat{F}_{\Theta^{l} \omega}^{l},
$$

and so

$$
E_{\hat{\mu}^{\omega}}\left(I_{0}^{(l)}(\omega)\right)^{2}=E_{\hat{\mu}^{\Theta l} \omega}\left(\hat{\Psi}_{\Theta^{l} \omega} E_{\hat{\mu}^{\Theta^{l} \omega}}\left(\hat{\Psi}_{\Theta^{l} \omega} \mid \hat{\mathcal{F}}_{-\infty, \Theta_{-l}\left(\Theta^{l} \omega\right)}\right)\right) .
$$

Since $\hat{\Psi}_{\Theta^{l} \omega}$ is $\hat{\mathcal{F}}_{0, \infty}^{\Theta^{l} \omega}$-measurable, in view of (4.1) I can apply to the right hand side of (4.3) Lemma 1 from Section 20 in [Bi1], which in the same way as in (3.7) yields that

$$
E_{\hat{\mu}^{\omega}}\left(I_{0}^{(l)}(\omega)\right)^{2} \leq 2 \phi_{l}^{1 / 2} C\left(\Theta^{l} \omega\right)\left(E_{\hat{\mu}^{\omega}}\left(I_{0}^{(l)}(\omega)\right)^{2}\right)^{1 / 2},
$$


and so

$$
\left(E_{\hat{\mu}^{\omega}}\left(I_{0}^{(l)}(\omega)\right)^{2}\right)^{1 / 2} \leq 2 \phi_{l}^{1 / 2} C\left(\Theta^{l} \omega\right) .
$$

Next, for $l<0$,

$$
J_{0}^{(l)}(\omega)=\left(\hat{\Psi}_{\Theta^{l} \omega}-E_{\hat{\mu}^{\Theta^{l} \omega}}\left(\hat{\Psi}_{\Theta^{l} \omega} \mid \hat{\mathcal{F}}_{-\infty, \Theta_{-l}\left(\Theta^{l} \omega\right)}^{\Theta^{\prime}}\right)\right) \circ\left(\hat{F}_{\Theta^{l} \omega}^{-l}\right)^{-1},
$$

and so

$$
E_{\hat{\mu}^{\omega}}\left(J_{0}^{(l)}\right)^{2}=D_{k_{-l}\left(\Theta^{l} \omega\right)}^{2}\left(\Theta^{l} \omega\right) .
$$

Now (4.4), (4.5) and similar estimates for $I_{1}^{(l)}$ and for $J_{1}^{(l)}$ together with (2.5) and (2.6) yield that for $P_{Q}-$ a.a. $\omega$ the series in (4.2) converge in $L^{2}\left(\hat{\Xi}^{\omega}, \hat{\mu}^{\omega}\right)$.

It is easy to see that $g_{\omega} \circ \hat{T}=\sum_{l=1}^{\infty} I_{0}^{(l)}-\sum_{l=-\infty}^{0} J_{0}^{(l)}(\omega)$, and so

$$
Y_{\omega}+g_{\omega}-(g \circ \hat{T})_{\omega}=I_{0}^{(0)}(\omega)+J_{0}^{(0)}=\hat{\Psi}_{\omega} .
$$

For $l=0,1, \ldots$ set $Y^{(l)}=Y \circ \hat{T}^{l}$; then $Y_{\omega}^{(l)}$ is $\hat{\mathcal{F}}_{-\infty, k_{l}(\omega)}^{\omega}$-measurable,

$$
\hat{\Psi} \circ \hat{T}^{l}=Y^{(l)}+g \circ \hat{T}^{l}-g \circ \hat{T}^{l+1},
$$

and

$$
E_{\hat{\mu}^{\omega}}\left(Y_{\omega}^{(l)} \mid \hat{\mathcal{F}}_{-\infty, k_{l-1}(\omega)}^{\omega}\right)=E_{\hat{\mu}^{\Theta^{l} \omega}}\left(Y_{\Theta^{l} \omega} \mid \hat{\mathcal{F}}_{-\infty, \Theta_{-1}\left(\Theta^{l} \omega\right)}^{\Theta^{l} \omega}\right) \circ \hat{F}_{\omega}^{l}=0,
$$

which provides the required martingale difference representation. Thus

$$
\frac{1}{\sqrt{m}} \sum_{l=0}^{m-1} \Psi \circ T^{l}=\frac{1}{\sqrt{m}} \sum_{l=0}^{m-1} Y^{(l)}+\frac{1}{\sqrt{m}}\left(g-g \circ T^{m}\right) .
$$

Since $g \in L^{2}\left(\hat{\Xi}_{Q}, \hat{\mu}_{Q}\right)$, where $\hat{\mu}_{Q}$ is the normalized restriction of $\hat{\mu}$ to $\hat{\Xi}_{Q}$, then the last term in the right hand side of (4.8) tends to 0 as $m \rightarrow \infty$ both $\mu_{Q}-$ a.s. and in $L^{2}\left(\hat{\Xi}^{\omega}, \hat{\mu}^{\omega}\right)$ for $P_{Q}-$ a.a. $\omega$. Note also that in view of $(2.7), \mu_{Q}-$ a.s. $\frac{1}{\sqrt{n}} R \circ T^{n} \rightarrow 0$ as $n \rightarrow \infty$. It follows that in order to prove Theorem 2.1 it suffices to derive the CLT and the invariance principle for the LIL for martingales $\sum_{l=0}^{m-1} Y_{\omega}^{(l)}$.

First, observe that by (4.6)-(4.8) and by the ergodic theorem, $P_{Q}-$ a.s.,

$$
\begin{aligned}
& \lim _{m \rightarrow \infty} \frac{1}{m} E_{\hat{\mu}^{\omega}}\left(\sum_{l=0}^{m-1}\left(\hat{\Psi} \circ T^{l}\right)_{\omega}\right)^{2}=\lim _{m \rightarrow \infty} \frac{1}{m} E_{\hat{\mu}^{\omega}}\left(\sum_{l=0}^{m-1} Y_{\omega}^{(l)}\right)^{2} \\
& =\lim _{m \rightarrow \infty} \frac{1}{m} \sum_{l=0}^{m-1} E_{\hat{\mu}^{\Theta^{l} \omega}}\left(Y_{\Theta^{l} \omega}\right)^{2}=E_{\hat{\mu}_{Q}}(\hat{\Psi}+g \circ \hat{T}-g)^{2} .
\end{aligned}
$$

This together with (3.16) yields that $\sigma=0$ if and only if $\hat{\Psi}=g-g \circ \hat{T} \hat{\mu}_{Q}-$ a.s.

Assume now that $\sigma>0$. In order to complete the CLT part of the proof of Theorem 2.1 by means of the central limit theorem for nonstationary martingale differences (see Section 4.1 in $[\mathrm{HH}]$ or $\S 8$ of Ch.VII from [Sh]) it remains only to verify the Lindenberg condition saying that, $P_{Q}-$ a.s.,

$$
\lim _{m \rightarrow \infty} \frac{1}{m} \sum_{l=0}^{m-1} E_{\mu^{\omega}}\left(\left(Y_{\omega}^{(l)}\right)^{2} \mathbb{I}_{\left|Y_{\omega}^{(l)}\right|>\varepsilon \sqrt{m}}\right)=0 .
$$


In order to derive (4.10) observe that by the ergodic theorem, $P_{Q}-$ a.s. and in $L^{1}\left(Q, P_{Q}\right)$ for any $K>0$,

$$
\begin{aligned}
& \limsup _{m \rightarrow \infty} \frac{1}{m} \sum_{l=0}^{m-1} E_{\hat{\mu}^{\omega}}\left(\left(Y \circ T^{l}\right)_{\omega}^{2} \mathbb{I}_{\left|\left(Y \circ T^{l}\right)_{\omega}\right|>\varepsilon \sqrt{m}}\right) \\
& =\limsup _{m \rightarrow \infty} \frac{1}{m} \sum_{l=0}^{m-1} E_{\hat{\mu}^{\Theta^{l} \omega}}\left(\left(Y_{\Theta^{l} \omega}\right)^{2} \mathbb{I}_{\left|Y_{\Theta^{l} \omega}\right|>\varepsilon \sqrt{m}}\right) \\
& \leq \lim _{m \rightarrow \infty} \frac{1}{m} \sum_{l=0}^{m-1} E_{\hat{\mu}^{\Theta^{l} \omega}}\left(\left(Y_{\Theta^{l} \omega}\right)^{2} \mathbb{I}_{\left|Y_{\Theta^{l} \omega}\right|>K}\right)=E_{P_{Q}} E_{\hat{\mu}^{\omega}}\left(\left(Y_{\omega}\right)^{2} \mathbb{I}_{\left|Y_{\omega}\right|>K}\right) .
\end{aligned}
$$

Since the last expression in (4.11) tends to zero as $K \rightarrow \infty$ and the first expression in (4.11) does not depend on $K$, then (4.10) follows.

In order to derive the invariance principle for the LIL for sums of the martingale differences $Y^{(l)}$ I shall employ Theorem 1 from [HS] (see also Section 4 in [HH]). The last condition of this theorem follows from (3.3), (3.4), (3.16) and (4.9), and the other two conditions hold true since

$$
\begin{aligned}
& E_{P_{Q}} \sum_{n=1}^{\infty} n^{-2} E_{\hat{\mu}^{\omega}}\left(\left(Y_{\omega}^{(n)}\right)^{4} \mathbb{I}_{\left|Y_{\omega}^{(n)}\right|<\delta n^{1 / 2}}\right)=E_{\hat{\mu}_{Q}}\left(Y^{4} \sum_{n=1}^{\infty} n^{-2} \mathbb{I}_{|Y|<\delta n^{1 / 2}}\right) \\
& \leq E_{\hat{\mu}_{Q}}\left(\delta^{4}+Y^{4} \int_{|Y|^{2} \delta^{-2}}^{\infty} x^{-2} d x\right)=\delta^{4}+\delta^{2} E_{\mu_{Q}} Y^{2}<\infty
\end{aligned}
$$

and

$$
\begin{aligned}
& E_{P_{Q}} \sum_{n=1}^{\infty} n^{-1 / 2} E_{\hat{\mu}^{\omega}}\left(\left|Y_{\omega}^{(n)}\right|{ }_{\omega} \mathbb{I}_{\left|Y_{\omega}^{(n)}\right| \geq \varepsilon n^{1 / 2}}\right)=E_{\hat{\mu}_{Q}}\left(|Y| \sum_{n=1}^{\infty} n^{-1 / 2} \mathbb{I}_{|Y| \geq \varepsilon n^{1 / 2}}\right) \\
& \leq E_{\hat{\mu}_{Q}}\left(|Y| \int_{0}^{|Y|^{2} \varepsilon^{-2}} x^{-1 / 2} d x\right)=2 \varepsilon^{-1} E_{\hat{\mu}_{Q}}|Y|^{2}<\infty
\end{aligned}
$$

Observe that, for any Borel set $U \subset \mathbb{R}$,

$$
\begin{aligned}
& \left\{\hat{\xi} \in \hat{\Xi}^{\omega}: \sum_{l=0}^{m-1} \hat{\Psi} \circ \hat{T}^{l}(\hat{\xi}, \omega) \in U\right\}=\left\{\hat{\xi} \in \hat{\Xi}^{\omega}: \sum_{l=0}^{m-1} \Psi_{\Theta^{l} \omega} \circ \iota_{\Theta^{l} \omega} \circ \hat{F}_{\omega}^{l}(\hat{\xi}) \in U\right\} \\
& =\left\{\hat{\xi} \in \hat{\Xi}^{\omega}: \sum_{l=0}^{m-1} \Psi_{\Theta^{l} \omega} \circ F_{\omega}^{l} \circ \iota_{\omega}(\hat{\xi}) \in U\right\}=\iota_{\omega}^{-1}\left(S_{\omega}^{(m)}\right)^{-1} U
\end{aligned}
$$

where $S_{\omega}^{(m)}=\sum_{l=0}^{m-1} \Psi_{\Theta^{l} \omega} \circ F_{\omega}^{l}$ is an $\mathcal{F}_{0, \infty}^{\omega}$-measurable function on $\Xi^{\omega}$. It follows that

$$
\begin{aligned}
& \hat{\mu}^{\omega}\left\{\hat{\xi} \in \hat{\Xi}^{\omega}: \sum_{l=0}^{m-1} \hat{\Psi} \circ \hat{T}^{l}(\hat{\xi}, \omega) \in U\right\}=\mu^{\omega}\left(\left(S_{\omega}^{(m)}\right)^{-1} U\right) \\
& =\mu^{\omega}\left\{\xi \in \Xi^{\omega}: \sum_{l=0}^{m-1} \Psi \circ T^{l}(\xi, \omega) \in U\right\} .
\end{aligned}
$$

Hence the CLT and the LIL derived above for $\sum_{l=0}^{m-1} \hat{\Psi} \circ \hat{T}^{l}$ with respect to $\hat{\mu}^{\omega}$ imply the CLT and the LIL for $\sum_{l=0}^{m-1} \Psi \circ T^{l}$ with respect to $\mu^{\omega}$, yielding Theorem 2.1 . 
4.1. Remark. It is possible to prove Theorem 2.1 and even stronger invariance principles without passing to random natural extension constructions as above by modifying methods from [PS] which lead to martingale difference representations of the form

$$
\Psi^{(N(n))} \circ T^{n}=Y^{(n)}+g^{(n)}-g^{(n+1)}
$$

where $\Psi_{\omega}^{(N)}=E_{\mu^{\omega}}\left(\Psi_{\omega} \mid \mathcal{F}_{0, k_{N}(\omega)}^{\omega}\right)$

$$
g_{\omega}^{(n)}=\sum_{l=0}^{\infty} E_{\mu^{\omega}}\left(\Psi^{(N(n+l))} \circ T^{n+l} \mid \mathcal{F}_{0, k_{n-1}(\omega)+N(n-1)}^{\omega}\right),
$$

and

$$
\begin{aligned}
Y_{\omega}^{n}=\sum_{l=0}^{\infty} & \left(E_{\mu^{\omega}}\left(\Psi^{(N(n+l))} \circ T^{n+l} \mid \mathcal{F}_{0, k_{n}(\omega)+N(n)}^{\omega}\right)\right. \\
& \left.-E_{\mu^{\omega}}\left(\Psi^{(N(n+l))} \circ T^{n+l} \mid \mathcal{F}_{0, k_{n}(\omega)+N(n-1)}^{\omega}\right)\right) .
\end{aligned}
$$

Here one chooses $N(j)$ to be small relative to $j^{1 / 2}$ and such that

$$
n^{-1 / 2} \sum_{j=0}^{n}\left(\Psi_{\omega}-\Psi_{\omega}^{(N(n))}\right) \circ T^{j} \rightarrow 0
$$

as $n \rightarrow \infty$. The corresponding estimates here are more technical than above, and they can be done similarly to [PS].

Next, I shall prove Theorem 2.3 working in the original spaces without using the above natural extension construction. Since (3.4) and (3.5) require only (2.7), which I assume also in Theorem 2.3, then one has to obtain only (3.16) and (3.17), which already yield (2.8) and (2.9). I shall find functions $Y_{\omega}^{(l)}=Y_{\omega}^{(l)}(\xi)$ and $g_{\omega}=g_{\omega}(\xi)$ belonging to $L^{2}\left(\Xi_{Q}, \mu_{Q}\right)$ such that for $P_{Q}-$ a.a. $\omega$ and any $l=1,2, \ldots$,

$$
E_{\mu^{\omega}}\left(Y_{\omega}^{(l)} \mid \mathcal{T}_{k_{l}(\omega)}^{\omega}\right)=0, \quad Y_{\omega}^{(l)} \text { is } \mathcal{T}_{k_{l-1}(\omega)}^{\omega}-\text { measurable }
$$

and

$$
\left(\Psi \circ T^{l}\right)_{\omega}=Y_{\omega}^{(l)}+\left(g \circ T^{l}\right)_{\omega}-\left(g \circ T^{l-1}\right)_{\omega},
$$

which will provide the required backwards martingale difference representation. If (4.15) holds true, then

$$
\frac{1}{\sqrt{m}} \sum_{l=1}^{m-1}\left(\Psi \circ T^{l}\right)_{\omega}=\frac{1}{\sqrt{m}} \sum_{l=1}^{m-1} Y_{\omega}^{(l)}+\frac{1}{\sqrt{m}}\left(\left(g \circ T^{m}\right)_{\omega}-g_{\omega}\right)+\frac{1}{\sqrt{m}} \Psi_{\omega}
$$

Since $g \in L^{2}\left(\Xi_{Q}, \mu_{Q}\right)$, the last two terms in the right hand side of (4.16) tend to zero as $m \rightarrow \infty$ in $L^{2}\left(\Xi^{\omega}, \mu^{\omega}\right)$ for $P_{Q}-$ a.a.w. Thus it will suffice to show that, $P_{Q}-$ a.s.,

$$
\lim _{m \rightarrow \infty} \frac{1}{m} E_{\mu^{\omega}}\left(\sum_{l=1}^{m-1} Y_{\omega}^{(l)}\right)^{2}=\lim _{m \rightarrow \infty} \frac{1}{m} E_{\mu^{\omega}}\left(\sum_{i=0}^{m-1}\left(\Psi \circ T^{i}\right)_{\omega}\right)^{2}=\sigma^{2} / P(Q)
$$

and to derive the $P$-a.s. CLT and LIL with respect to $\mu^{\omega}$ for backwards martingales $\sum_{l=1}^{m-1} Y_{\omega}^{(l)}$. 
Set

$$
g_{\omega}=\sum_{n=0}^{\infty} U_{\Theta}^{* n} \Psi_{\Theta^{-n} \omega}
$$

then by the assumption (ii) of Theorem $2.3 \mathrm{~g}$ belongs to $L^{2}\left(\Xi_{Q}, \mu_{Q}\right)$. Clearly,

$$
\Psi_{\Theta \omega}=g_{\Theta \omega}-U_{\omega}^{*} g_{\omega} .
$$

Observe that for any bounded $\mathcal{T}_{0}^{\Theta \omega}$-measurable function $q$ on $\Xi^{\Theta \omega}$,

$$
E_{\mu^{\ominus \omega}}\left(q U_{\omega}^{*} E_{\mu}^{\omega}\left(g_{\omega} \mid \mathcal{T}_{k_{1}(\omega)}^{\omega}\right)\right)=E_{\mu^{\omega}}\left(\left(U_{\omega} q\right) g_{\omega}\right)=E_{\mu^{\ominus \omega}}\left(q U_{\omega}^{*} g_{\omega}\right),
$$

since $U_{\omega} q$ is $\mathcal{T}_{k_{1}(\omega)}^{\omega}$-measurable. Hence $U_{\omega}^{*} g_{\omega}=U_{\omega}^{*} E_{\mu}^{\omega}\left(g_{\omega} \mid \mathcal{T}_{k_{1}(\omega)}^{\omega}\right) \mu^{\Theta \omega}$-a.s., which together with (4.19) gives

$$
\Psi_{\Theta \omega}=g_{\Theta \omega}-U_{\omega}^{*} E_{\mu}^{\omega}\left(g_{\omega} \mid \mathcal{T}_{k_{1}(\omega)}^{\omega}\right) .
$$

Applying $U_{\omega}$ to both parts of (4.21) and taking into account that $U_{\omega} U_{\omega}^{*} q=q$ for any $\mathcal{T}_{k_{1}(\omega)}^{\omega}$-measurable $q$, I obtain

$$
\Psi_{\Theta \omega} \circ F_{\omega}=g_{\Theta \omega} \circ F_{\omega}-E_{\mu^{\omega}}\left(g_{\omega} \mid \mathcal{T}_{k_{1}(\omega)}^{\omega}\right) .
$$

Considering (4.22) for $\Theta^{l-1} \omega$ in place of $\omega$ and applying $U_{\omega}^{l-1}$ to both parts, I derive

$$
\begin{aligned}
& \Psi_{\Theta^{l} \omega} \circ F_{\omega}^{l}=g_{\Theta^{l} \omega} \circ F_{\omega}^{l}-E_{\mu^{\Theta^{l-1} \omega}}\left(g_{\Theta^{l-1} \omega} \mid \mathcal{T}_{k_{1}(\omega)}^{\Theta^{l-1} \omega}\right) \circ F_{\omega}^{l-1} \\
& =\left(g \circ T^{l}\right)_{\omega}-E_{\mu^{\omega}}\left(\left(g \circ T^{l-1}\right)_{\omega} \mid \mathcal{T}_{k_{l}(\omega)}^{\omega}\right) .
\end{aligned}
$$

Taking conditional expectations in (4.23), I have

$$
E_{\mu^{\omega}}\left(\left(\Psi \circ T^{l}\right)_{\omega} \mid \mathcal{T}_{k_{l}(\omega)}^{\omega}\right)=E_{\mu^{\omega}}\left(\left(g \circ T^{l}\right)_{\omega} \mid \mathcal{T}_{k_{l}(\omega)}^{\omega}\right)-E_{\mu^{\omega}}\left(\left(g \circ T^{l-1}\right)_{\omega} \mid \mathcal{T}_{k_{l}(\omega)}^{\omega}\right) .
$$

Set $Y_{\omega}^{(l)}=\left(\Psi \circ T^{l}\right)_{\omega}-\left(g \circ T^{l}\right)_{\omega}+\left(g \circ T^{l-1}\right)_{\omega}$. Then (4.24) implies (4.14) and

$$
Y_{\omega}^{(l)}=\left(Y^{(1)} \circ T^{l-1}\right)_{\omega} .
$$

In fact, one can reverse the above arguments and conclude that such $g_{\omega}$ and $Y_{\omega}^{(l)}$ are the only possible choices for the functions satisfying (4.14) and (4.15).

Next I shall prove (4.17). It follows from assumption (i) of Theorem 2.3 that the series (3.10) converges absolutely $P_{Q}-$ a.s. and in $L^{1}\left(Q, P_{Q}\right)$. One has

$$
\left|\Phi\left(\Theta^{l} \omega\right)-\Phi_{m-l}\left(\Theta^{l} \omega\right)\right| \leq \tilde{\delta}_{m-l}\left(\Theta^{l} \omega\right),
$$

where

$$
\tilde{\delta}_{l}(\omega)=\sum_{j=l}^{\infty}\left|E_{\mu^{\omega}}\left(\Psi_{\omega}\left(\Psi \circ T^{l}\right)_{\omega}\right)\right| .
$$

In the same way as in (3.14)-(3.15) I derive (3.16) from this and assumption (i) of Theorem 2.3. Thus the second equality in (4.17) holds true, and the first equality there follows from (4.16) and the fact that

$$
\lim _{n \rightarrow \infty} \frac{1}{n} E_{\mu^{\omega}}\left(g \circ T^{n}\right)_{\omega}^{2}=\lim _{n \rightarrow \infty} \frac{1}{n} E_{\mu^{\Theta^{n}}} g_{\Theta^{n} \omega}^{2}=0,
$$

since $E_{\mu}^{\omega} g_{\omega} \in L^{2}\left(Q, P_{Q}\right)$. Observe that by (4.14), for any $l_{2}>l_{1} \geq 1$,

$$
E_{\mu^{\omega}}\left(Y_{\omega}^{\left(l_{1}\right)} Y_{\omega}^{\left(l_{2}\right)}\right)=E_{\mu^{\omega}}\left(Y_{\omega}^{\left(l_{2}\right)} E_{\mu^{\omega}}\left(Y_{\omega}^{\left(l_{1}\right)} \mid \mathcal{T}_{k_{l_{1}}(\omega)}^{\omega}\right)\right)=0,
$$


and so, by (4.15), (4.25) and the ergodic theorem, $P_{Q}-$ a.s.,

$$
\begin{aligned}
& \lim _{m \rightarrow \infty} \frac{1}{m} E_{\mu^{\omega}}\left(\sum_{l=1}^{m-1} Y_{\omega}^{(l)}\right)^{2}=\lim _{m \rightarrow \infty} \frac{1}{m} \sum_{l=1}^{m-1} E_{\mu^{\omega}}\left(Y_{\omega}^{(l)}\right)^{2} \\
& =\lim _{m \rightarrow \infty} \frac{1}{m} \sum_{l=1}^{m-1} E_{\mu^{\Theta^{l} \omega}}\left(Y_{\Theta^{l} \omega}^{(1)}\right)^{2}=E_{\mu_{Q}}(\Psi \circ T-g \circ T+g)^{2} .
\end{aligned}
$$

This together with (4.17) yields that $\sigma=0$ if and only if $\Psi \circ T=g \circ T-g$ $\mu_{Q}-$ a.s. Finally, assuming that $\sigma>0$, I verify the Lindenberg condition (4.10) for martingale differences $Y_{\omega}^{(l)}$ required for application of the CLT results from [HH] and $[\mathrm{Sh}]$ and the conditions for the LIL from [HS] and $[\mathrm{HH}]$ exactly in the same way as in (4.11)-(4.13) above, which completes the proof of Theorem 2.3.

\section{MARKOV CHAINS IN RANDOM ENVIRONMENTS}

In this section I shall derive Theorem 2.4 from Theorem 2.1, verifying the conditions of the latter. Define inductively $\tilde{k}_{i+1}(\omega)=\min \left\{k>\tilde{k}_{i}(\omega): \theta^{-k} \omega \in Q\right\}$ with $\tilde{k}_{0}(\omega) \equiv 0$ and $\tilde{n}_{1}=\tilde{n}_{1}(\omega)=\min \left\{\tilde{k}_{i}(\omega): \tilde{k}_{i}(\omega) \geq L\right\}$ and $\tilde{n}_{j+1}(\omega)=\min \left\{\tilde{k}_{i}(\omega):\right.$ $\left.\tilde{k}_{i}(\omega) \geq \tilde{n}_{j}(\omega)+L\right\}, j=1,2, \ldots . \operatorname{Set} \tilde{l}^{\omega}(n)=\tilde{l}_{L}^{\omega}(n)=\max \left\{j: \tilde{n}_{j}(\omega)<n\right\}$. By $(2.12)$ from [Ki3] it follows that for any $x \in X^{\theta^{-n} \omega}$, a measurable $\Gamma \subset X^{\omega}$, and $P$-a.a. $\tilde{\omega} \in \Omega$,

$$
\left|P^{\theta^{-n} \tilde{\omega}}(n, x, \Gamma)-\eta^{\tilde{\omega}}(\Gamma)\right| \leq 2\left(1-L^{-1}\right)^{\tilde{l}^{\tilde{\omega}}(n)},
$$

where $\eta^{\omega}$ is the unique probability measure satisfying (2.15). Take $\tilde{\omega}=\theta^{k_{i+j}(\omega)} \omega$, $n=k_{i+j}(\omega)-k_{i}(\omega)=k_{j}\left(\theta^{k_{i}(\omega)} \omega\right)$, and set

$$
\zeta_{n}^{x, \omega}(\Gamma)=P^{\omega}(n, x, \Gamma)-\eta^{\theta^{n} \omega}(\Gamma)
$$

Then by (5.1), for any $y \in X^{\theta^{k_{i}(\omega)} \omega}$ and a measurable $\Gamma \subset X^{\theta^{k_{i+j}(\omega)} \omega}$,

$$
\left|\zeta_{k_{i+j}(\omega)-k_{i}(\omega)}^{y, \theta^{k_{i}(\omega)}}(\Gamma)\right| \leq 2\left(1-L^{-1}\right)^{\tilde{l}^{k^{k_{i+j}(\omega)} \omega}\left(k_{i+j}(\omega)-k_{i}(\omega)\right)} \leq 2\left(1-L^{-1}\right)^{j / L} .
$$

In order to verify (2.5) it suffices to consider the right hand side of (2.2) for $A \in \mathcal{F}_{0, k_{i}(\omega)}^{\omega}$ of the form $A=\left\{\xi: Z_{1}^{\omega}(\xi) \in \Gamma_{1}, \ldots, Z_{k_{i}(\omega)}^{\omega}(\xi) \in \Gamma_{k_{i}(\omega)}\right\}$ and an arbitrary $B \in \mathcal{F}_{k_{i+j}(\omega), \infty}^{\omega}$. By (2.14), (2.15), (5.2), (5.3), and the Markov property it follows that

$$
\begin{aligned}
& \mu^{\omega}(A \cap B)=\int_{X^{\omega}} d \eta^{\omega}(x) \int_{\Gamma_{1}} \cdots \int_{\Gamma_{k_{i}(\omega)}} P^{\omega}\left(x, d z_{1}\right) \cdots P^{\theta^{k_{i}(\omega)-1} \omega}\left(z_{k_{i}(\omega)-1}, d z_{k_{i}(\omega)}\right) \\
& \times \int_{X^{\theta^{k_{i+j}(\omega)} \omega}} P^{\theta^{k_{i}(\omega)} \omega}\left(k_{i+j}(\omega)-k_{i}(\omega), z_{k_{i}}, d v\right) \mu^{\omega}\left(B \mid Z_{k_{i+j}(\omega)}^{\omega}=v\right) \\
& =\mu^{\omega}(A) \mu^{\omega}(B)+I_{A, B}^{\omega},
\end{aligned}
$$

where

$$
\begin{aligned}
& \left|I_{A, B}^{\omega}\right| \leq \sup _{y \in X^{\theta^{k_{i}(\omega)} \omega}}\left|\int_{X^{\theta^{k_{i+j}(\omega)} \omega}} \zeta_{k_{i+j}(\omega)-\theta_{i}(\omega)}^{y, \theta^{k_{i}(\omega) \omega}}(d v) \mu^{\omega}\left(B \mid Z_{k_{i+j}(\omega)}^{\omega}=v\right)\right| \\
& \leq \sup _{y \in X^{\theta^{k_{i}(\omega)} \omega}} \zeta_{k_{i+j}(\omega)-\theta_{i}(\omega)}^{y, \theta^{k_{i}(\omega)} \omega}\left(\Gamma_{y}^{+}\right) \leq 2\left(1-L^{-1}\right)^{j / L}
\end{aligned}
$$


and $\Gamma_{y}^{+} \subset X^{\theta^{k_{i+j}(\omega)} \omega}$ is the positive set in the Hahn decomposition (see [Bi2]) for the signed measure $\zeta_{k_{i+j}(\omega)-k_{i}(\omega)}^{y, \theta_{i}(\omega)}$. Hence

$$
\phi_{j} \leq 2\left(1-L^{-1}\right)^{j / L}
$$

and so (2.5) holds true. Thus if (2.6) and (2.7) are satisfied, then (2.8) and (2.9) follow by Theorem 2.1. If $\varphi_{\omega}(\xi)=\varphi_{\omega}\left(Z^{\omega}(\xi)\right)$ is, in fact, a function on $X$ and $\psi_{\omega}(z)=\varphi_{\omega}(z)-E_{\mu^{\omega}} \varphi_{\omega}\left(Z^{\omega}(\xi)\right)=\varphi_{\omega}(z)-E_{\eta^{\omega}} \varphi_{\omega}$, then the second equality in (2.17) follows from Theorem 2.1.

In order to obtain the first equality in $(2.17)$, set

$$
S_{n}^{\omega}(\xi)=\sum_{k=0}^{n-1} \psi\left(Z_{k}^{\omega}(\xi), \theta^{k} \omega\right), \quad S_{m, n}^{\omega}(\xi)=S_{n}^{\omega}(\xi)-S_{m}^{\omega}(\xi) \text { for } n \geq m
$$

$\mu_{x}^{\omega}\{\cdot\}=\mu^{\omega}\left\{\cdot \mid Z_{0}^{\omega}=x\right\}$, and observe that

$$
\begin{aligned}
& \mu_{x}^{\omega}\left\{\xi: \frac{1}{\sqrt{n}} S_{k_{i}(\omega), n}^{\omega}(\xi) \leq a-\delta\right\}-\mu_{x}^{\omega}\left\{\xi: \frac{1}{\sqrt{n}}\left|S_{0, k_{i}(\omega)}^{\omega}(\xi)\right|>\delta\right\} \\
& \leq \mu_{x}^{\omega}\left\{\xi: \frac{1}{\sqrt{n}} S_{0, n}^{\omega}(\xi) \leq a\right\} \\
& \leq \mu_{x}^{\omega}\left\{\xi: \frac{1}{\sqrt{n}} S_{k_{i}(\omega), n}^{\omega}(\xi) \leq a+\delta\right\}+\mu_{x}^{\omega}\left\{\xi: \frac{1}{\sqrt{n}}\left|S_{0, k_{i}(\omega)}^{\omega}(\xi)\right|>\delta\right\} .
\end{aligned}
$$

By (2.14), (2.15), (5.2), (5.3), and the Markov property it follows that

$$
\begin{aligned}
& \mu_{x}^{\omega}\left\{\xi: \frac{1}{\sqrt{n}} S_{k_{i}(\omega), n}^{\omega}(\xi) \leq b\right\} \\
& +\int_{X^{\theta^{k_{i}(\omega)} \omega}} P^{\omega}\left(k_{i}(\omega), x, d y\right) \mu^{\omega}\left\{\xi: \frac{1}{\sqrt{n}} S_{k_{i}(\omega), n}^{\omega}(\xi) \leq b \mid Z_{k_{i}(\omega)}^{\omega}=y\right\} \\
& =\mu^{\omega}\left\{\xi: \frac{1}{\sqrt{n}} S_{k_{i}(\omega), n}^{\omega}(\xi) \leq b\right\}+J_{x, i}^{\omega},
\end{aligned}
$$

where

$$
\left|J_{x, i}^{\omega}\right| \leq \sup _{x \in X^{\omega}} \zeta_{k_{i}(\omega)}^{x, \omega}\left(\tilde{\Gamma}_{x}^{+}\right) \leq 2\left(1-L^{-1}\right)^{i / L}
$$

and $\tilde{\Gamma}_{x}^{+}$is the positive set in the Hahn decomposition for the signed measure $\zeta_{k_{i}(\omega)}^{x, \omega}$. Letting, first, $n \rightarrow \infty$, then $i \rightarrow \infty$, and finally $\delta \rightarrow 0$, I conclude from (5.7)-(5.10) that both limits in (2.17) are the same, provided the second limit in (2.17) exists and equals the right hand side of (2.17), which I have proved already.

\section{RANDOM SUBSHIFTS AND RANDOM EXPANDING TRANSFORMATIONS}

In this section I shall verify the conditions of Theorem 2.1 for random subshifts of finite type and of Theorem 2.3 for random expanding in average transformations, extending to my more general situation the deterministic arguments from Lemmas 1.9-1.12 and 1.14 in [Bo]. Whenever possible I shall deal with both cases simultaneously. Set

$$
R_{\omega}=\sum_{l=1}^{\infty} K_{g}\left(\theta^{-l} \omega\right) e^{-\kappa l}, \quad \rho(\xi, \tilde{\xi})=e^{-\min \left\{j \geq 0: \xi_{j} \neq \tilde{\xi}_{j}\right\}}
$$


provided $\xi, \tilde{\xi} \in \Xi^{\omega}$, in the case of random subshifts,

$$
R_{\omega}=\sum_{l=1}^{\infty} K_{g}\left(\theta^{-l} \omega\right)\left(\left\|D f_{\theta^{-l} \omega}^{-1}\right\| \cdots\left\|D f_{\theta^{-2} \omega}^{-1}\right\|\left\|D f_{\theta^{-1} \omega}^{-1}\right\|\right)^{\kappa}, \rho(\xi, \tilde{\xi})=d(\xi, \tilde{\xi})
$$

in the case of random expanding transformations, and in both cases

$$
R_{\omega}(\xi, \tilde{\xi})=R_{\omega}(\rho(\xi, \tilde{\xi}))^{\kappa}
$$

By (2.21) and (2.29) it follows that $R_{\omega}<\infty P$-a.s.

In the expanding random transformations case set

$$
Z_{\omega}=\sum_{l=1}^{\infty}\left\|D f_{\theta^{-l} \omega}^{-1}\right\| \cdots\left\|D f_{\theta^{-2} \omega}^{-1}\right\|\left\|D f_{\theta^{-1} \omega}^{-1}\right\|
$$

and $\varepsilon_{\omega}=\frac{1}{2} \min \left(1, \rho_{M}\right) \min \left(1, Z_{\omega}^{-1}\right)$, where $\rho_{M}$ is the injectivity radius of the Riemannian manifold $M$. It follows (see Exercise 2.3 and its solution in Ch. 2 of [Ha]) that, for any $z \in M$, if $f_{\omega}^{-1} z=\left\{z_{1}, \ldots, z_{m}\right\}$ then the balls $B_{z_{i}}\left(\frac{\varepsilon_{\theta \omega}}{\left\|D f_{\omega}\right\|}\right)$ are disjoint and there exist closed domains $U_{z_{i}} \supset B_{z_{i}}\left(\frac{\varepsilon_{\theta \omega}}{\left\|D f_{\omega}\right\|}\right)$ which are mapped by $f_{\omega}$ diffeomorphically onto $B_{z}\left(\varepsilon_{\theta \omega}\right)$, and so each $U_{z_{i}}$ may contain only one preimage of each point from $B_{z}\left(\varepsilon_{\theta \omega}\right)$. In particular, distances between different preimages under $f_{\omega}$ of any point in $M$ are not less than $\frac{\varepsilon_{\theta \omega}}{\left\|D f_{\omega}\right\|}$, and so the number $\ell_{\omega}$ of preimages under $f_{\omega}$ of any point of $M$ is bounded by $C\left(\frac{\varepsilon_{\theta \omega}}{\left\|D f_{\omega}\right\|}\right)^{d}$, where $C>0$ depends only on $M$. If $d(z, \tilde{z}) \leq \varepsilon_{\theta \omega}$, then for each $i$ there is a unique $\tilde{z}_{i} \in f_{\omega}^{-1} \tilde{z}$ belonging to $U_{z_{i}}$ and

$$
\begin{aligned}
& d\left(z_{i}, \tilde{z}_{i}\right) \leq\left\|D f_{\omega}^{-1}\right\| d(z, \tilde{z}) \leq \frac{1}{2} \min \left(1, \rho_{M}\right) \min \left(\left\|D f_{\omega}^{-1}\right\|, \frac{\left\|D f_{\omega}^{-1}\right\|}{Z_{\theta \omega}}\right) \\
& \leq \frac{1}{2} \min \left(1, \rho_{M}\right)\left(1+Z_{\omega}\right)^{-1} \leq \varepsilon_{\omega} .
\end{aligned}
$$

In order to deal simultaneously both with random expanding transformations and with random subshifts I set also in the second case $\varepsilon_{\omega}=e^{-1}$ and define in both cases a family of cones of continuous functions on $\Xi^{\omega}$ by

$$
\Lambda_{\gamma}^{\omega}=\left\{q: q \geq 0, \int q d \nu^{\omega}=1 \text { and } q(\xi) \leq e^{\gamma R_{\omega}(\xi, \tilde{\xi})} q(\tilde{\xi}) \text { if } \rho(\xi, \tilde{\xi}) \leq \varepsilon_{\omega}\right\},
$$

where $\nu^{\omega}$ is the same as in (2.25) and $\gamma \geq 1$ is a constant. By (2.25) and (6.4) it is easy to check directly (cf. [Ki1]) that

$$
\lambda_{\omega}^{-1} \mathcal{L}_{g}^{\omega} \Lambda_{\gamma}^{\omega} \subset \Lambda_{\gamma}^{\theta \omega} \text { for any } \gamma \geq 1
$$

where $\lambda_{\omega}$ is the same as in (2.25), and by the construction, the functions $h_{\omega}$ from (2.25) belong to $\Lambda_{1}^{\omega}$. (see $\left.[\mathrm{KK}]\right)$. It is easy to see that there exists a constant $\tilde{C}>0$ depending only on $M$ such that for any $C^{1}$ endomorphism $f: M \rightarrow M$ and any $x \in M$ the set $f^{-1} x$ is $\tilde{C}\left\|D f^{-1}\right\|$-net, i.e. for any $y \in M$ there exists $z \in f^{-1} x$ such that $d(y, z) \leq \tilde{C}\left\|D f^{-1}\right\|$. Applying this to $f_{\omega}^{n}$ and taking into account that, by (2.29) and the ergodic theorem,

$$
\lim _{n \rightarrow \infty} \frac{1}{n} \log \left\|\left(D f_{\omega}^{n}\right)^{-1}\right\| \leq \lim _{n \rightarrow \infty} \frac{1}{n} \sum_{j=0}^{n-1} \log \left\|D f_{\theta^{j} \omega}^{-1}\right\|=\alpha<0 P-\text { a.s. }
$$

I conclude that there exists a random variable $N=N_{\omega}<\infty$ on $(\Omega, \mathcal{G}, P)$ with values in $\mathbb{Z}^{+}$such that $P$-a.s. for any $x \in M$ the set $\left(f_{\omega}^{N}\right)^{-1} x$ is an $\varepsilon_{\omega}-$ net. In the case of random subshifts I denote by $N=N_{\omega}$ a $\mathbb{Z}^{+}$-valued random variable such 
that $A(\omega) A(\theta \omega) \cdots A\left(\theta^{n} \omega\right)$ is a positive matrix for all $n \geq N$, where the $A(\omega)$ 's are $\ell(\omega) \times \ell(\theta \omega)$ matrices apearing in the definition of a random subshift in Section 2 . Such an $N_{\omega}$ exists by the topological mixing assumption in Theorem 2.5. Observe that this definition of $N=N_{\omega}$ is equivalent to saying that for $P$-a.a. $\omega$ and any $\eta \in \Xi^{\theta^{N} \omega}$ the set $\left(f_{\omega}^{N}\right)^{-1} \eta$ is an $\varepsilon(\omega)-$ net in $\Xi^{\omega}$ with respect to the metric $\rho(\cdot, \cdot)$ defined in (6.1), since both characterizations of $N_{\omega}$ are equivalent to the third one saying that for any $j \in\{1, \ldots, \ell(\omega)\}$ the set $\left(f_{\omega}^{N}\right)^{-1}$ contains a point $\xi=\left(\xi_{0}, \xi_{1}, \ldots\right)$ from $\Xi^{\omega}$ with $\xi_{0}=j$. Thus the characterization of $N_{\omega}$ is, essentially, the same in both cases under consideration, which enables me to give their simultaneous treatment.

I claim that in both cases, for any $q \in \Lambda_{\gamma}^{\omega}$,

$$
\inf _{\xi}\left(\lambda_{\omega} \cdots \lambda_{\theta^{N_{\omega}-1} \omega}\right)^{-1} \mathcal{L}_{g}^{\omega, N_{\omega}} q(\xi) \geq G_{\omega}^{-1} e^{-\gamma H_{\omega}^{(1)}}
$$

where $G_{\omega}=\lambda_{\omega} \cdots \lambda_{\theta^{N_{\omega}-1} \omega} \exp \left(\sum_{j=0}^{N_{\omega}-1}\left\|g_{\theta^{j} \omega}\right\|\right)$ with $\|\cdot\|$ being the supremum norm, $H_{\omega}^{(a)}(\omega)=R_{\omega} \varepsilon_{\omega}^{a}, \mathcal{L}_{g}^{\omega, n}=\mathcal{L}_{g}^{\theta^{n-1} \omega} \circ \cdots \circ \mathcal{L}_{g}^{\theta \omega} \circ \mathcal{L}_{g}^{\omega}$, and I take the infinum in (6.8) over all $\xi \in \Xi^{\theta^{N \omega} \omega}$ where in the random expanding transformations case $\Xi^{\omega}=M$ for all $\omega \in \Omega$. Indeed, if $\xi \in \Xi^{\theta^{N} \omega}, \eta \in \Xi^{\omega}$ then there exists $\zeta^{\prime} \in\left(f_{\omega}^{N}\right)^{-1} \xi \in \Xi^{\omega}$ with $\rho\left(\eta, \zeta^{\prime}\right) \leq \varepsilon_{\omega}$, and so by $(2.24)$,

$$
\begin{aligned}
& \mathcal{L}_{g}^{\omega, N_{\omega}} q(\xi)=\sum_{\zeta \in\left(f_{\omega}^{N \omega}\right)^{-1} \xi} \exp \left(\sum_{j=0}^{N_{\omega}-1} g \circ \tau^{j}(\zeta, \omega)\right) q(\zeta) \\
& \geq e^{-\sum_{j=0}^{N_{\omega}-1}\left\|g_{\theta j}\right\|_{\omega}} q\left(\zeta^{\prime}\right) \geq e^{-\sum_{j=0}^{N_{\omega}-1}\left\|g_{\theta^{j}}\right\|} e^{-\gamma R_{\omega} \varepsilon_{\omega}} q(\eta) .
\end{aligned}
$$

Since $\int_{\Xi^{\omega}} q d \nu^{\omega}=1$ and $q \geq 0$, then there exists $\eta \in \Xi^{\omega}$ with $q(\eta) \geq 1$, and for such $\eta$ (6.9) implies (6.8). Since $\int \mathcal{L}_{g}^{\omega, N_{\omega}} q d \nu^{\theta^{N_{\omega}} \omega}=\lambda_{\omega} \cdots \lambda_{\theta^{N_{\omega}-1} \omega}$, (6.9) yields also that for any $q \in \Lambda_{\gamma}^{\omega}$,

$$
q \leq G_{\omega} e^{\gamma H_{\omega}^{(1)}}
$$

Next I shall need

6.1. Lemma. For P-a.a.w and any $\gamma \geq 2$ there exists $a_{\omega}=a_{\omega, \gamma} \leq \frac{1}{2}$ such that for any $\gamma \geq 2$ and $q \in \Lambda_{\gamma}^{\omega}$ one can find $q^{\prime} \in \Lambda_{\gamma}^{\theta^{N_{\omega} \omega}}$ satisfying

$$
\left(\lambda_{\omega} \cdots \lambda_{\theta^{N \omega-1} \omega}\right)^{-1} \mathcal{L}_{g}^{\omega, N_{\omega}} q=a_{\omega} h_{\theta^{N_{\omega}} \omega}+\left(1-a_{\omega}\right) q^{\prime},
$$

where $h_{\omega}$ is the same as in (2.25).

Proof. I have to choose $a_{\omega}$ so that

$$
\left(\lambda_{\omega} \cdots \lambda_{\theta^{N_{\omega}-1} \omega}\right)^{-1} \mathcal{L}_{g}^{\omega, N_{\omega}} q-a_{\omega} h_{\theta^{N_{\omega} \omega}} \in \Lambda_{\gamma}^{\theta^{N_{\omega}} \omega},
$$

which requires

$$
\begin{aligned}
& \left(\lambda_{\omega} \cdots \lambda_{\theta^{N_{\omega}-1} \omega}\right)^{-1} \mathcal{L}_{g}^{\omega, N_{\omega}} q(\xi)-a_{\omega} h_{\theta^{N_{\omega} \omega}}(\xi) \\
& \leq e^{\gamma R_{\theta^{N} \omega}(\xi, \tilde{\xi})}\left(\left(\lambda_{\omega} \cdots \lambda_{\theta^{N_{\omega}-1} \omega}\right)^{-1} \mathcal{L}_{g}^{\omega, N_{\omega}} q(\tilde{\xi})-a_{\omega} h_{\theta^{N_{\omega} \omega}}(\tilde{\xi})\right),
\end{aligned}
$$


provided $\rho(\xi, \tilde{\xi}) \leq \varepsilon_{\theta^{N_{\omega} \omega}}$, or, which is the same,

$$
\begin{aligned}
& a_{\omega}\left(e^{\gamma R_{\theta^{N} \omega}(\xi, \tilde{\xi})} h_{\theta^{N_{\omega} \omega}}(\tilde{\xi})-h_{\theta^{N} \omega \omega}(\xi)\right) \\
& \leq e^{\gamma R_{\theta^{N} \omega}(\xi, \tilde{\xi})}\left(\lambda_{\omega} \cdots \lambda_{\theta^{N_{\omega}-1} \omega}\right)^{-1} \mathcal{L}_{g}^{\omega, N_{\omega}} q(\tilde{\xi})-\left(\lambda_{\omega} \cdots \lambda_{\theta^{N_{\omega}-1} \omega}\right)^{-1} \mathcal{L}_{g}^{\omega, N_{\omega}} q(\xi) .
\end{aligned}
$$

By (6.6),

$$
\tilde{q} \stackrel{\text { def }}{=}\left(\lambda_{\omega} \cdots \lambda_{\theta^{N_{\omega}-2} \omega}\right)^{-1} \mathcal{L}_{g}^{\omega, N_{\omega}-1} q \in \Lambda_{\gamma}^{\theta^{N_{\omega}-1} \omega} .
$$

Observe that if $\xi, \tilde{\xi} \in \Xi^{\theta^{N \omega} \omega}$ satisfy $\rho(\xi, \tilde{\xi}) \leq \varepsilon_{\theta^{N} \omega \omega}$, then there exists a one-to-one correspondence between points $\xi^{\prime} \in f_{\theta^{N_{\omega}-1} \omega}^{-1} \xi$ and $\tilde{\xi}^{\prime} \in f_{\theta^{N_{\omega}-1} \omega}^{-1} \tilde{\xi}$ so that

$$
\rho\left(\xi^{\prime}, \tilde{\xi}^{\prime}\right) \leq \min \left(\varepsilon_{\theta^{N_{\omega}-1} \omega}, D_{\theta^{N_{\omega}-1} \omega} \rho(\xi, \tilde{\xi})\right),
$$

where $D_{\omega}=e^{-1}$ for random subshifts and $D_{\omega}=\left\|D f_{\omega}^{-1}\right\|$ for random expanding transformations. Set $D_{\omega}(\xi, \tilde{\xi})=K_{g}(\omega) D_{\omega}^{\kappa}(\rho(\xi, \tilde{\xi}))^{\kappa}$; then

$$
\gamma D_{\theta^{N_{\omega}-1} \omega}(\xi, \tilde{\xi})+D_{\theta^{N_{\omega}-1} \omega}^{\kappa} R_{\theta^{N \omega}-1}(\xi, \tilde{\xi})=R_{\theta^{N_{\omega} \omega}}(\xi, \tilde{\xi}),
$$

and so by $(2.22),(2.30),(6.14)$, and $(6.15)$, for any $\xi, \tilde{\xi} \in \Xi^{\theta^{N \omega} \omega}$ as above

$$
\begin{aligned}
& \left(\lambda_{\theta^{N} \omega-1} \omega\right)^{-1} \mathcal{L}_{g}^{\theta^{N_{\omega}-1}} \tilde{q}(\xi)=\left(\lambda_{\theta^{N} \omega-1}\right)^{-1} \sum_{\xi^{\prime} \in f_{\theta^{N} \omega-1}^{-1} \xi} e^{g_{\theta^{N} \omega_{\omega}-1}\left(\xi^{\prime}\right)} \tilde{q}\left(z^{\prime}\right) \\
& \leq e^{-(\gamma-1) D_{\theta^{N} \omega-1}(\xi, \tilde{\xi})+\gamma R_{\theta^{N} \omega}(\xi, \tilde{\xi})}\left(\lambda_{\theta^{N} \omega-1}\right)^{-1} \mathcal{L}_{g}^{\theta^{N_{\omega}-1} \omega} \tilde{q}(\tilde{\xi}) .
\end{aligned}
$$

So in order to obtain (6.13) it suffices to have

$$
\begin{aligned}
& a_{\omega}\left(e^{\gamma R_{\theta^{N \omega}}(\xi, \tilde{\xi})} h_{\theta^{N_{\omega} \omega}}(\tilde{\xi})-h_{\theta^{N_{\omega} \omega}}(\xi)\right) \\
& \leq\left(e^{\gamma R_{\theta^{N} \omega_{\omega}}(\xi, \tilde{\xi})}-e^{-(\gamma-1) D_{\theta^{N_{\omega}-1} \omega}(\xi, \tilde{\xi})+\gamma R_{\theta^{N_{\omega}}}(\xi, \tilde{\xi})}\right)\left(\lambda_{\omega} \cdots \lambda_{\theta^{N_{\omega}-1} \omega}\right)^{-1} \mathcal{L}_{g}^{\omega, N_{\omega}} q(\tilde{\xi}) .
\end{aligned}
$$

Since by the construction $h_{\theta^{N_{\omega} \omega}} \in \Lambda_{1}^{\theta^{N_{\omega}} \omega}$, and so $h_{\theta^{N_{\omega} \omega}}(\tilde{\xi}) \leq e^{R_{\theta^{N_{\omega}}}(\xi, \tilde{\xi})} h_{\theta^{N_{\omega} \omega}}(\xi)$, then taking into account (6.8) and (6.10) (the latter for $q=h_{\theta^{N_{\omega} \omega}}$ ) it suffices to obtain that

$$
\begin{aligned}
& a_{\omega}\left(e^{\gamma R_{\theta^{N} \omega}(\xi, \tilde{\xi})}-e^{-R_{\theta^{N} \omega_{\omega}}(\xi, \tilde{\xi})}\right) G_{\theta^{N \omega} \omega} e^{H_{\theta^{N} \omega \omega}^{(1)}} \\
& \leq\left(e^{\gamma R_{\theta N_{\omega} \omega}(\xi, \tilde{\xi})}-e^{-(\gamma-1) D_{\theta^{N} \omega-1}(\xi, \tilde{\xi})+\gamma R_{\theta N_{\omega}}(\xi, \tilde{\xi})}\right) G_{\omega}^{-1} e^{-H_{\omega}^{(1)}} .
\end{aligned}
$$

Next, for $\xi, \tilde{\xi}$ as above,

$$
0 \leq R_{\theta^{N_{\omega} \omega}}(\xi, \tilde{\xi}) \leq R_{\omega} \varepsilon_{\omega}^{\kappa}=H_{\omega}^{(\kappa)}
$$

and

$$
0 \leq \gamma R_{\theta^{N \omega} \omega}(\xi, \tilde{\xi})-(\gamma-1) D_{\theta^{N_{\omega}-1} \omega}(\xi, \tilde{\xi}) \leq \gamma R_{\theta^{N \omega \omega}}(\xi, \tilde{\xi}) .
$$

For any $x, y \in\left[-H_{\omega}^{(\kappa)}, \gamma H_{\omega}^{(\kappa)}\right]$ one has

$$
e^{-H_{\omega}^{(\kappa)}}(x-y) \leq e^{x}-e^{y} \leq e^{\gamma H_{\omega}^{(\kappa)}}(x-y) .
$$


Hence in order to obtain (6.16) it suffices to have

$$
(\gamma+1) a_{\omega} e^{(\gamma+1) H_{\theta^{N} \omega \omega}^{(\kappa)}} R_{\theta^{N} \omega \omega}(\xi, \tilde{\xi}) G_{\theta^{N} \omega \omega} \leq(\gamma-1) D_{\theta^{N} \omega-1}(\xi, \tilde{\xi}) G_{\omega}^{-1} e^{-\gamma H_{\omega}^{(1)}}
$$

and since

$$
D_{\theta^{N_{\omega}-1} \omega}(\xi, \tilde{\xi}) R_{\theta^{N_{\omega} \omega}}=R_{\theta^{N_{\omega} \omega}}(\xi, \tilde{\xi}) K_{g}\left(\theta^{N_{\omega}-1} \omega\right) D_{\theta^{N_{\omega}-1} \omega}^{\kappa}
$$

and I always can assume that $K_{g} \geq 1$, I only need that

$$
a_{\omega} \leq \frac{1}{3} e^{-\gamma\left(2 H_{\theta^{N} \omega}^{(\kappa)}+H_{\omega}^{(1)}\right)} \Upsilon_{\omega}
$$

where $\Upsilon_{\omega}=G_{\theta^{N \omega} \omega}^{-1} G_{\omega}^{-1} D_{\theta^{N \omega}-1}^{\kappa} R_{\theta^{N \omega} \omega}^{-1}$. Observe that in view of (6.1), (6.2), (6.8), and (6.10) the inequality (6.17) implies that $a_{\omega} h_{\theta^{N \omega} \omega} \leq\left(\lambda_{\omega} \cdots \lambda_{\theta^{N \omega}-1}\right)^{-1} \mathcal{L}_{g}^{\omega, N_{\omega}} q$, i.e. the other condition for (6.12) is also satisfied. Thus, defining $a_{\omega}$ by the right hand side of (6.17), I complete the proof of Lemma 6.1.

Let $Q$ be a measurable set in $\Omega$ such that $P(Q)>0$ and

$$
Q \subset Q_{L}=\left\{\omega: \max \left(N_{\omega}, 2 H_{\theta^{N \omega} \omega}^{(\kappa)}+H_{\omega}^{(1)}, G_{\omega}, \Upsilon_{\omega}^{-1}\right) \leq L\right\},
$$

and define the hitting times $k_{i}(\omega)$ by $(2.3)$.

6.2. Lemma. For any $\omega \in Q, q \in \Lambda_{\gamma}^{\omega}, \gamma \geq 2$, and $n$ satisfying $k_{j}(\omega) \leq n$ one has

$$
\left\|\left(\lambda_{\omega} \cdots \lambda_{\theta^{n} \omega}\right)^{-1} \mathcal{L}_{g}^{\omega, n} q-h_{\theta^{n} \omega}\right\| \leq 2 L e^{\gamma L}\left(1-\frac{1}{3} L^{-1} e^{-\gamma L}\right)^{[j / L]} .
$$

Proof. It follows from (6.11) that for any $\tilde{\omega}=\theta^{k_{i}(\omega)} \omega, q \in \Lambda_{\gamma}^{\tilde{\omega}}$, and $l \geq L$ there exists $q^{\prime} \in \Lambda_{\gamma}^{\theta^{l} \omega}$ such that

$$
\left(\lambda_{\tilde{\omega}} \cdots \lambda_{\theta^{l-1} \tilde{\omega}}\right)^{-1} \mathcal{L}_{g}^{\tilde{\omega}, l} q=a_{\tilde{\omega}} h_{\theta^{l} \tilde{\omega}}+\left(1-a_{\tilde{\omega}}\right) q^{\prime} .
$$

By induction it is easy to derive from (6.20) that for any $i=1,2, \ldots$ and $q \in \Lambda_{\gamma}^{\tilde{\omega}}$ there exists $q_{i L}^{\prime} \in \Lambda_{\gamma}^{\theta^{k_{i L}(\omega)} \omega}$ such that

$$
\begin{aligned}
& \left(\lambda_{\omega} \cdots \lambda_{\theta^{k_{i L}(\omega)-1} \omega}\right)^{-1} \mathcal{L}_{g}^{\omega, k_{i L}(\omega)} q=\left(1-\prod_{l=0}^{i-1}\left(1-a_{\theta^{k_{l L}(\omega)} \omega}\right)\right) h_{\theta^{k_{i L}(\omega)} \omega} \\
& +q_{i L}^{\prime} \prod_{l=0}^{i-1}\left(1-a_{\theta^{k_{l L}(\omega)} \omega}\right) .
\end{aligned}
$$

This together with (6.10), (6.18), and the choice of $a_{\omega}$ yields (6.19).

6.3. Lemma. Let $q$ and $r$ be measurable functions on $\Xi^{\omega}$ and $\Xi^{\theta^{k_{j}(\omega)} \omega}$, respectively, such that $\|q\|,\|r\|<\infty$ and there exists $K_{q} \geq 1$ with

$$
|q(\xi)-q(\tilde{\xi})| \leq K_{q}(\rho(\xi, \tilde{\xi}))^{\kappa} \forall \xi, \tilde{\xi} \in \Xi^{\omega}
$$

where $k_{j}(\omega)$ are the same as in Lemma 6.2. Then

$$
\begin{aligned}
& \left|\int q\left(r \circ f_{\omega}^{k_{j}(\omega)}\right) d \mu^{\omega}-\int q d \mu^{\omega} \int r d \mu^{\theta^{k_{j}(\omega)} \omega}\right| \\
& \leq 4 L e^{2 L}\left(\|q\|+K_{q} R_{\omega}^{-1}\right)\|r\|\left(1-\frac{1}{3} L^{-1} e^{-2 L}\right)^{[j / L]}
\end{aligned}
$$


and

$$
\begin{aligned}
& \left\|\left(\lambda_{\omega} \cdots \lambda_{\theta^{k_{j}(\omega)} \omega}\right)^{-1} h_{\theta^{k_{j}(\omega)} \omega}^{-1} \mathcal{L}_{g}^{\omega, k_{j}(\omega)} q-\int q d \mu^{\omega}\right\|, \\
& \leq 4 L e^{2 L}\left(\|q\|+K_{q} R_{\omega}^{-1}\right)\left\|h_{\theta^{k_{j}(\omega)} \omega}^{-1}\right\|\left(1-\frac{1}{3} L^{-1} e^{-2 L}\right)^{[j / L]},
\end{aligned}
$$

where $\mu^{\omega}$ is the same as in (2.26) and $\gamma=\gamma_{\omega}=1+K_{q} R_{\omega}^{-1}$.

Proof. Observe that, by (2.28),

$$
R_{\omega} \geq K_{g}\left(\theta^{-1} \omega\right)\left\|D f_{\theta^{-1} \omega}^{-1}\right\| \geq\left\|D f_{\theta^{-1} \omega}^{-1}\right\| \geq\left\|D f_{\theta^{-1} \omega}\right\|^{-1}>0 P \text { - a.s. }
$$

since I assume (without loss of generality) that $K_{g}(\omega) \geq 1$. Suppose, first, that $q \geq 0$, and set $\hat{q}=\left(q+K_{q} R_{\omega}^{-1}\right)\left(\int q d \mu^{\omega}+K_{q} R_{\omega}^{-1}\right)^{-1}$. Then $\int \hat{q} h_{\omega} d \nu^{\omega}=1$ and, by (6.21), for any $\xi, \tilde{\xi} \in \Xi^{\omega}$,

$$
\hat{q}(\xi) \leq \hat{q}(\tilde{\xi})\left(1+\frac{K_{q}(\rho(\xi, \tilde{\xi}))^{\kappa}}{q(\tilde{\xi})+K_{q} R_{\omega}^{-1}}\right) \leq \hat{q}(\tilde{\xi})\left(1+R_{\omega}(\xi, \tilde{\xi})\right) \leq \hat{q}(\tilde{\xi}) e^{R_{\omega}(\xi, \tilde{\xi})},
$$

and since $h_{\omega} \in \Lambda_{1}^{\omega}$ I derive that $q h_{\omega} \in \Lambda_{2}^{\omega}$. Thus by (2.27) and (6.19),

$$
\begin{aligned}
& \left|\int q\left(r \circ f_{\omega}^{k_{j}(\omega)}\right) d \mu^{\omega}-\int q d \mu^{\omega} \int r d \mu^{\theta^{k_{j}(\omega)} \omega}\right| \\
& =\left(\int q d \mu^{\omega}+\frac{K_{q}}{R_{\omega}}\right)\left|\int \hat{q}\left(r \circ f_{\omega}^{k_{j}(\omega)}\right) h_{\omega} d \nu^{\omega}-\int r d \mu^{\theta^{k_{j}(\omega)} \omega}\right| \\
& =\left(\int q d \mu^{\omega}+\frac{K_{q}}{R_{\omega}}\right) \\
& \times\left|\left(\lambda_{\omega} \cdots \lambda_{\theta^{k_{j}(\omega)} \omega}\right)^{-1} \int r \mathcal{L}_{g}^{\omega, k_{j}(\omega)}\left(\hat{q} h_{\omega}\right) d \nu^{\theta^{k_{j}(\omega)} \omega}-\int r h_{\theta^{k_{j}(\omega)} \omega} d \nu^{\theta^{k_{j}(\omega)} \omega}\right| \\
& \leq\left(\|q\|+K_{q} R_{\omega}^{-1}\right)\|r\|\left\|\left(\lambda_{\omega} \cdots \lambda_{\theta^{k_{j}(\omega)} \omega}\right)^{-1} \mathcal{L}_{g}^{\omega, k_{j}(\omega)}\left(\hat{q} h_{\omega}\right)-h_{\theta^{k_{j}(\omega)} \omega}\right\| \\
& \leq 2 L e^{2 L}\left(\|q\|+K_{q} R_{\omega}^{-1}\right)\|r\|\left(1-\frac{1}{3} L^{-1} e^{-2 L}\right)^{[j / L]},
\end{aligned}
$$

proving (6.22) for a nonnegative $q$. Similarly, by (6.19),

$$
\begin{aligned}
& \left\|\left(\lambda_{\omega} \cdots \lambda_{\theta^{k_{j}(\omega)} \omega}\right)^{-1} h_{\theta^{k_{j}(\omega)} \omega}^{-\mathcal{L}_{g}} \mathcal{L}_{g, k_{j}(\omega)} q-\int q d \mu^{\omega}\right\| \\
& \times\left\|h_{\theta^{k_{j}(\omega)} \omega}^{-1}\right\|\left\|\left(\lambda_{\omega} \cdots \lambda_{\theta^{k_{j}(\omega)} \omega}\right)^{-1} \mathcal{L}_{g}^{\omega, k_{j}(\omega)} \hat{q}-h_{\theta^{k_{j}(\omega)} \omega}\right\| \\
& \leq 2 L e^{2 L}\left(\|q\|+K_{q} R_{\omega}^{-1}\right)\left\|h_{\theta^{k_{j}(\omega)} \omega}^{-1}\right\|\left(1-\frac{1}{3} L^{-1} e^{-2 L}\right)^{[j / L]},
\end{aligned}
$$

proving (6.23) for a nonnegative $q$. For a general $q$, represent it as the difference of its positive $q_{+}$and negative $q_{-}$parts and apply (6.26) and (6.27) to each of them; then (6.22) and (6.23) follow via the triangle inequality.

I shall deal first with the random expanding maps case. In order to employ the estimate (6.23) I first need an upper bound for $\left\|h_{\omega}^{-1}\right\|$. Observe that as $n \rightarrow \infty$,

$$
\begin{aligned}
& \left\|\left(D f_{\theta^{-n} \omega}^{n}\right)^{-1}\right\| Z_{\theta^{-n} \omega} \leq\left\|D f_{\theta^{-n} \omega}^{-1}\right\| \cdots\left\|D f_{\theta^{-1} \omega}^{-1}\right\| Z_{\theta^{-n} \omega} \\
& =\sum_{l=n+1}^{\infty}\left\|D f_{\theta^{-l} \omega}^{-1}\right\| \cdots\left\|D f_{\theta^{-1} \omega}^{-1}\right\| \rightarrow 0
\end{aligned}
$$


as the remainder of a converging series. It follows that there exists a random variable $\tilde{N}=\tilde{N}_{\omega}$ such that $\forall x \in M$ the set $\left(f_{\theta^{-\tilde{N}_{\omega}}}^{\tilde{N}}\right)^{-1} x$ is an $\varepsilon_{\theta^{-\tilde{N}_{\omega}}}-$ net. Thus for any $x, y \in M$ there exists $z \in\left(f_{\theta^{-\tilde{N}} \omega}^{\tilde{N}}\right)^{-1} x$ with $d(y, z) \leq \varepsilon_{\theta^{-\tilde{N}} \omega}$, and so

$$
\begin{aligned}
& \lambda_{\theta^{-\tilde{N}} \omega} \cdots \lambda_{\theta^{-1} \omega} h_{\omega}(x)=\mathcal{L}_{g}^{\theta^{-\tilde{N}} \omega, \tilde{N}} h_{\theta^{-\tilde{N}} \omega}(x) \geq h_{\theta^{-\tilde{N}} \omega}(z) \exp \left(-\sum_{j=1}^{\tilde{N}}\left\|g_{\theta^{-j} \omega}\right\|\right) \\
& \geq h_{\theta^{-\tilde{N}} \omega} \exp \left(-\gamma R_{\theta^{-\tilde{N}} \omega} \varepsilon_{\theta^{-\tilde{N}} \omega}-\sum_{j=1}^{\tilde{N}}\left\|g_{\theta^{-j} \omega}\right\|\right) .
\end{aligned}
$$

Since $\int h_{\theta^{-\tilde{N}} \omega} d \nu^{\theta^{-\tilde{N}} \omega}=1$, I can choose $y$ so that $h_{\theta^{-\tilde{N}} \omega}(y) \geq 1$. Taking into account also that

$$
\lambda_{\theta^{-\tilde{N}} \omega} \cdots \lambda_{\theta^{-1} \omega}=\int \mathcal{L}_{g}^{\theta^{-\tilde{N}} \omega, \tilde{N}} 1 d \nu^{\omega} \leq \prod_{j=1}^{\tilde{N}} \ell\left(\theta^{-j} \omega\right) e^{\left\|g_{\theta^{-j} \omega}\right\|}
$$

I derive that for any $x \in M$,

$$
h_{\omega}(x) \geq e^{-\gamma R_{\theta^{-\tilde{N}_{\omega}}} \varepsilon_{\theta}-\tilde{N}_{\omega}} \prod_{j=1}^{\tilde{N}} \ell^{-1}\left(\theta^{-j} \omega\right) e^{-2\left\|g_{\theta-j}\right\|} \stackrel{\text { def }}{=} \iota(\omega),
$$

and so $\left\|h_{\omega}^{-1}\right\| \leq \iota^{-1}(\omega)$.

Now take $Q=Q_{L} \cap\left\{\omega: \max \left(R_{\omega}^{-1}, \iota^{-1}(\omega)\right) \leq L\right\}$ with $Q_{L}$ defined by (6.18), and apply (6.22) and (6.23) with $q=\Psi_{\omega}$ and $r=\Psi_{\theta^{k_{j}(\omega)} \omega}$. Since $\int \Psi_{\omega} d \mu^{\omega}=$ $\int \Psi_{\theta^{k_{j}(\omega)} \omega} d \mu^{\theta^{k_{j}(\omega)} \omega}=0$, it follows by (2.31), (6.22), and the Cauchy-Schwarz inequality that

$$
E_{P_{Q}} \sum_{n=0}^{\infty}\left|E_{\mu^{\omega}}\left(\Psi_{\omega}\left(\Psi \circ T^{n}\right)_{\omega}\right)\right| \leq 12 L^{4} e^{4 L} E_{P_{Q}}\left(\left\|\Psi_{\omega}\right\|+K_{\Psi}(\omega)\right)^{2}<\infty,
$$

establishing condition (i) of Theorem 2.3. In order to derive condition (ii) I use the formula

$$
u_{\omega}^{*} q_{\omega}=\lambda_{\omega}^{-1} h_{\theta \omega}^{-1} \mathcal{L}_{g}^{\omega}\left(q_{\omega} h_{\omega}\right)
$$

which holds true since for any $q \in L^{2}\left(\Xi^{\omega}, \mu^{\omega}\right)$ and $r \in L^{2}\left(\Xi^{\theta \omega}, \mu^{\theta \omega}\right)$,

$$
\begin{aligned}
& \lambda_{\omega}^{-1} \int r h_{\theta \omega}^{-1} \mathcal{L}_{g}^{\omega}\left(q h_{\omega}\right) d \mu^{\theta \omega}=\lambda_{\omega}^{-1} \int r \mathcal{L}_{g}^{\omega}\left(q h_{\omega}\right) d \nu^{\theta \omega} \\
& =\lambda_{\omega}^{-1} \int \mathcal{L}_{g}^{\omega}\left(q h_{\omega} u_{\omega} r\right) d \nu^{\theta \omega}=\int\left(u_{\omega} r\right) q d \mu^{\omega} .
\end{aligned}
$$

Applying (6.29) successively, I obtain that

$$
U_{\Theta^{-n} \omega}^{* n} \Psi_{\Theta^{-n} \omega}=\left(\lambda_{\tilde{\omega}} \cdots \lambda_{\theta^{k_{n}(\tilde{\omega})} \tilde{\omega}}\right)^{-1} h_{\theta^{k_{n}(\tilde{\omega})} \tilde{\omega}}^{-1} \mathcal{L}_{g}^{\tilde{\omega}, k_{n}(\tilde{\omega})} \Psi_{\tilde{\omega}} \text {, with } \tilde{\omega}=\Theta^{-n} \omega .
$$


Therefore by (2.31), (6.23), and the Cauchy-Schwarz inequality,

$$
\begin{aligned}
E_{P_{Q}} & E_{\mu^{\omega}}\left(\sum_{n=0}^{\infty}\left|U_{\Theta^{-n} \omega}^{* n} \Psi_{\Theta^{-n} \omega}\right|\right)^{2} \\
& \leq 16 L^{4} e^{4 L} E_{P_{Q}}\left(\sum_{n=0}^{\infty}\left(\left\|\Psi_{\Theta^{-n} \omega}\right\|+K_{\Psi}\left(\Theta^{-n} \omega\right)\right)\left(1-\frac{1}{3} L^{-1} e^{-2 L}\right)^{[n / L]}\right)^{2} \\
& \leq 48 L^{6} e^{6 L} E_{P_{Q}} \sum_{n=0}^{\infty}\left(\| \Psi_{\Theta^{-n}} \omega+K_{\Psi}\left(\Theta^{-n} \omega\right)\right)^{2}\left(1-\frac{1}{3} L^{-1} e^{-2 L}\right)^{[n / L]} \\
& =144 L^{8} e^{8 L} E_{P_{Q}}\left(\left\|\Psi_{\omega}\right\|+K_{\Psi}(\omega)\right)^{2}<\infty
\end{aligned}
$$

establishing the condition (ii) of Theorem 2.3, and so completing the proof of Theorem 2.6.

In order to complete the proof of Theorem 2.5 I shall need a few additional arguments which can be applied only in the random subshifts case. Denote by $\mathcal{C}_{n}^{\omega}$ the set of functions $r$ on $\Xi^{\omega}$ such that $\operatorname{var}_{n}^{\omega} r=0$, with $\operatorname{var}_{n}^{\omega}$ defined by $(2.20)$.

6.4. Lemma. If $q \in \Lambda_{\gamma}^{\omega}, \gamma \geq 1, r \in \mathcal{C}_{n}^{\omega}, r \geq 0$, and $r q \not \equiv 0$, then

$$
\left(\int q r d \nu^{\omega}\right)^{-1}\left(\lambda_{\omega} \cdots \lambda_{\theta^{n-1} \omega}\right)^{-1} \mathcal{L}_{g}^{\omega, n}(q r) \in \Lambda_{\gamma}^{\theta^{n} \omega} .
$$

Proof. Let $\xi, \tilde{\xi} \in \Xi^{\omega}$ and $\rho(\xi, \tilde{\xi})=e^{-m}$, then

$$
\begin{aligned}
& \mathcal{L}_{g}^{\omega, n}(q r)(\xi)=\sum_{\zeta \in\left(f_{\omega}^{n}\right)^{-1} \xi} \exp \left(\sum_{j=0}^{n-1} g \circ \tau^{j}(\zeta, \omega)\right) q(\zeta) r(\zeta) \\
& \leq \sum_{\tilde{\zeta} \in\left(f_{\omega}^{n}\right)^{-1} \tilde{\xi}} \exp \left(\sum_{j=0}^{n-1}\left(g \circ \tau^{j}(\tilde{\zeta}, \omega)+\operatorname{var}_{m+n-j}^{\theta^{j} \omega} g\right)\right) e^{\gamma R_{\omega}(\zeta, \tilde{\zeta})} q(\tilde{\zeta}) r(\tilde{\zeta}),
\end{aligned}
$$

where $\rho(\zeta, \tilde{\zeta})=e^{-(m+n)}$. By $(2.22),(6.1)$, and (6.3),

$$
\begin{aligned}
& R_{\omega}(\zeta, \tilde{\zeta})+\sum_{j=0}^{n-1} \operatorname{var}_{m+n-j}^{\theta^{j} \omega} g \leq \sum_{j=1}^{\infty} K_{g}\left(\theta^{-j} \omega\right) e^{-\kappa(m+n+j)} \\
& +\sum_{l=0}^{n-1} K_{g}\left(\theta^{l} \omega\right) e^{-\kappa(m+n-l)}=R_{\theta^{n} \omega}(\xi, \tilde{\xi}) .
\end{aligned}
$$

This together with (6.32) yields that for $\gamma \geq 1$,

$$
\mathcal{L}_{g}^{\omega, n}(q r)(\xi) \leq e^{\gamma R_{\theta^{n} \omega}(\xi, \tilde{\xi})} \mathcal{L}_{g}^{\omega, n}(q r)(\tilde{\xi}) .
$$

Next, in the same way as in (6.9),

$$
\begin{aligned}
& \int q r d \nu^{\omega} \\
& =\left(\lambda_{\theta^{n} \omega} \cdots \lambda_{\theta^{N^{n} \omega^{-1}}\left(\theta^{n} \omega\right)}\right)^{-1} \int \mathcal{L}_{g}^{\theta^{n} \omega, N_{\theta^{n} \omega}}\left(\lambda_{\omega} \cdots \lambda_{\theta^{n-1} \omega}\right)^{-1} \mathcal{L}_{g}^{\omega, n}(q r) d \nu^{\omega} \\
& \geq G_{\omega}^{-1} e^{-\gamma B_{1}(\omega)}\left(\lambda_{\omega} \cdots \lambda_{\theta^{n-1} \omega}\right)^{-1} \mathcal{L}_{g}^{\omega, n}(q r)(\xi)
\end{aligned}
$$

for any $\xi \in \Xi^{\theta^{n} \omega}$. Since $q r \not \equiv 0$, there exists $\xi$ so that the right hand side of (6.34) is not equal to 0 , and so the left hand side of (6.34) is positive. This together with (6.33) yields (6.31). 
Now I can complete the proof of Theorem 2.5. Take $Q=Q_{L}$ with $Q_{L}$ defined by (6.18), and consider cylinder sets $A=\left\{\xi=\left(\xi_{0}, \xi_{1}, \ldots\right) \in \Xi^{\omega}: \xi_{i}=a_{i}\right.$ for $i=$ $0,1, \ldots, l\}$ and $B=\left\{\zeta=\left(\zeta_{0}, \zeta_{1}, \ldots\right) \in \Xi^{\theta^{l+n} \omega}: \zeta_{i}=b_{i}\right.$ for $\left.i=0,1, \ldots, m\right\}$. Then

$$
\begin{aligned}
& \mu^{\omega}\left(A \cap\left(f_{\omega}^{l+n}\right)^{-1} B\right)=\int \mathbb{I}_{A}\left(\mathbb{I}_{B} \circ f_{\omega}^{l+n}\right) h_{\omega} d \nu^{\omega} \\
& =\left(\lambda_{\omega} \cdots \lambda_{\theta^{l+n-1} \omega}\right)^{-1} \int \mathbb{I}_{B} \mathcal{L}_{g}^{\omega, l+n}\left(\mathbb{I}_{A} h_{\omega}\right) d \nu^{\theta^{l+n} \omega} .
\end{aligned}
$$

Since $\mathbb{I}_{A} \in \mathcal{C}_{l}^{\omega}, \mathbb{I}_{A} \geq 0, h_{\omega} \in \Lambda_{1}^{\omega}$, and $h_{\omega}>0$, then by Lemma 6.4 ,

$$
q_{\theta^{l} \omega} \stackrel{\text { def }}{=}\left(\int h_{\omega} \mathbb{I}_{A} d \nu^{\omega}\right)^{-1}\left(\lambda_{\omega} \cdots \lambda_{\theta^{l} \omega}\right)^{-1} \mathcal{L}_{g}^{\omega, l}\left(\mathbb{I}_{A} h_{\omega}\right) \in \Lambda_{1}^{\theta^{l} \omega},
$$

and so by (6.35) and by (6.19) considered with $\tilde{\omega}=\theta^{l} \omega$ in place of $\omega$ and $n \geq k_{j}(\tilde{\omega})$ it follows that

$$
\begin{aligned}
& \left|\mu^{\omega}\left(A \cap\left(f_{\omega}^{l+n}\right)^{-1} B\right)-\mu^{\omega}(A) \mu^{\theta^{l+n} \omega}(B)\right| \\
& =\mid \int\left(\left(\lambda_{\omega} \cdots \lambda_{\theta^{l+n-1} \omega}\right)^{-1} \mathcal{L}_{g}^{\omega, l+n}\left(\mathbb{I}_{A} h_{\omega}\right)\right. \\
& \left.-\left(\int h_{\omega} \mathbb{I}_{A} d \nu^{\omega}\right) h_{\theta^{l+n} \omega}\right) \mathbb{I}_{B} d \nu^{\theta^{l+n} \omega} \mid \\
& \leq \mu^{\omega}(A) \nu^{\theta^{l+n} \omega}(B)\left\|\left(\lambda_{\tilde{\omega}} \cdots \lambda_{\theta^{n} \tilde{\omega}}\right)^{-1} \mathcal{L}_{g}^{\tilde{\omega}, n} q_{\tilde{\omega}}-h_{\theta^{n} \tilde{\omega}}\right\| \\
& \leq 2 \mu^{\omega}(A) \nu^{\theta^{l+n} \omega}(B) L e^{L}\left(1-\frac{1}{3} L^{-1} e^{-L}\right)^{[j / L]} .
\end{aligned}
$$

Take $l=k_{i}(\omega)$ and $n=k_{j}\left(\theta^{l} \omega\right)$; then, by the choice of $Q=Q_{L}, \nu^{\theta^{l+n} \omega}(B) \leq$ $\mu^{\theta^{l+n} \omega}(B)\left\|h_{\theta^{l+n} \omega}^{-1}\right\| \leq L \mu^{\theta^{l+n} \omega}(B)$, which together with (6.36) yields (2.5). This yields (2.5) and completes the proof of Theorem 2.5.

6.5. Remark. It is possible to define the set $Q$ via the function $g$ and intrinsic characterictics of random subshifts and random expanding transformations. In order to do that it is necessary to obtain appropriate upper bounds for $G_{\omega}$ and $\left\|h_{\omega}\right\|$. Since $h_{\omega} \in \Lambda_{1}^{\omega}$, then by (6.10) one needs only an upper bound on $G_{\omega}$. In view of the definition of $G_{\omega}$ one has to obtain an upper bound for $\lambda_{\omega} \cdots \lambda_{\theta^{N_{\omega}-1} \omega}$, which follows by

$$
\lambda_{\omega} \cdots \lambda_{\theta^{N \omega}-1} \omega=\int \mathcal{L}_{g}^{\omega, N_{\omega}} 1 d \nu^{\theta^{N_{\omega}} \omega} \leq \prod_{j=0}^{N_{\omega}-1} \ell\left(\theta^{j} \omega\right) \exp \left(\sum_{j=0}^{N_{\omega}-1}\left\|g_{\theta^{j} \omega}\right\|\right)
$$

Thus I can take $Q=\tilde{Q}_{L}=\left\{\omega: \max \left(N_{\omega}, \tilde{G}_{\omega}, \tilde{G}_{\theta^{N_{\omega} \omega}}, R_{\omega}, R_{\theta^{N_{\omega} \omega}}\right) \leq L\right\}$ with $\tilde{G}_{\omega}=\prod_{j=0}^{N_{\omega}-1} \ell\left(\theta^{j} \omega\right) e^{\left\|g_{\theta^{j}}\right\|}$ in the random subshift case, and $Q=\tilde{Q}_{L} \cap\{\omega$ : $\left.\max \left(R_{\omega}^{-1}, \iota^{-1}(\omega)\right) \leq L\right\}$ in the random expanding maps case.

\section{RANDOM SUSPENSIONS}

In order to prove Theorem 2.8, set $\bar{l}_{\omega}=\int l_{\omega}(\xi) d \mu^{\omega}(\xi)$ and apply Theorem 2.1 to the function $l$. Then for any $\alpha>0, P-$ a.s.,

$$
\lim _{n \rightarrow \infty} \mu^{\omega}\left\{\xi: n^{-\left(\frac{1}{2}+\alpha\right)}\left|\sum_{i=0}^{n-1}\left(l_{\theta^{i} \omega}-\bar{l}_{\theta^{i} \omega}\right)\left(f_{\theta^{i} \omega}^{i}(\xi)\right)\right|>\delta\right\}=0 \forall \delta>0 .
$$


Set $t_{\omega}(k)=\sum_{i=0}^{k} \bar{l}_{\theta^{i} \omega}$ and $n_{\omega}(\xi, s)=\max \left\{n \geq 0: \sum_{i=0}^{n}\left(l \circ \tau^{i}\right)_{\omega}(\xi) \leq s\right\}$. Then

$$
\begin{aligned}
& \left\{\xi:\left|n_{\omega}\left(\xi, t_{\omega}(k)\right)-k\right| \geq b \sqrt{k}\right\}=\left\{\xi: \sum_{0 \leq i \leq k+b \sqrt{k}}\left(l \circ \tau^{i}\right)_{\omega}(\xi) \leq \sum_{k=0}^{k} \bar{l}_{\theta^{i} \omega}\right\} \\
& \cup\left\{\xi: \sum_{0 \leq i \leq j}\left(l \circ \tau^{i}\right)_{\omega}(\xi)>\sum_{i=0}^{k} \bar{l}_{\theta^{i} \omega} \text { for some } j=0, \ldots,[k-b \sqrt{k}]+1\right\} \\
& \subset\left\{\xi: k^{-1 / 2}\left|\sum_{i=0}^{k}\left(l_{\theta^{i} \omega}-\bar{l}_{\theta^{i} \omega}\right)\left(f_{\omega}^{i} \xi\right)\right| \geq(b-1) C_{1}^{-1}\right\},
\end{aligned}
$$

where $C_{1}=\sup _{\omega} C(\omega)$. By (7.1) and (7.2), P-a.s. for any $\alpha>0$,

$$
\lim _{k \rightarrow \infty} \mu^{\omega}\left\{\xi:\left|n_{\omega}\left(\xi, t_{\omega}(k)\right)-k\right|>k^{\frac{1}{2}+\alpha}\right\}=0 .
$$

Observe that

$$
\int_{0}^{t}\left(\psi \circ \sigma^{s}\right)_{\omega}(0, \xi) d s=\sum_{i=0}^{n_{\omega}(\xi, t)}\left(\psi^{(l)} \circ \tau^{i}\right)_{\omega}(\xi)+\int_{s_{\omega}(\xi, t)}^{t}\left(\psi \circ \sigma^{s}\right)_{\omega}(0, \xi) d s,
$$

where $s_{\omega}(\xi, t)=\sum_{i=0}^{n_{\omega}(\xi, t)}\left(l \circ \tau^{i}\right)_{\omega}(\xi)$ and $0 \leq t-s_{\omega}(\xi, t) \leq C_{1}$, and

$$
\left|\int_{s_{\omega}(\xi, t)}^{t}\left(\psi \circ \sigma^{s}\right)_{\omega}(0, \xi) d s\right| \leq\left(q \circ \tau^{n_{\omega}(\xi, t)}\right)_{\omega}(\xi),
$$

where by assumption

$$
q_{\omega}(\xi)=\int_{0}^{l_{\omega}(\xi)}\left|\psi_{\omega}(\xi, s)\right| d s \in L^{1}\left(\Xi_{Q}, \mu_{Q}\right)
$$

In view of (7.3)-(7.6) it is easy to see that in order to obtain (2.35) it suffices to show that, $P$-a.s. for any $a$,

$$
\lim _{k \rightarrow \infty} \mu^{\omega}\left\{\xi \in \Xi^{\omega}: \frac{1}{\sqrt{k}} \sum_{i=0}^{k}\left(\psi^{(l)} \circ \tau^{i}\right)_{\omega}(\xi) \leq a\right\}=\frac{1}{\tilde{\sigma} \sqrt{2 \pi}} \int_{-\infty}^{a} e^{-\frac{x^{2}}{2 \tilde{\sigma}^{2}}} d x,
$$

where

$$
\begin{aligned}
& \tilde{\sigma}^{2}=\lim _{k \rightarrow \infty} \frac{1}{k} E_{\mu^{\omega}}\left(\sum_{i=0}^{k-1}\left(\psi^{(l)} \circ \tau^{i}\right)_{\omega}\right)^{2} \\
& =P(Q)\left(E_{\mu_{Q}}\left(\Psi^{(l)}\right)^{2}+\sum_{j=1}^{\infty} E_{\mu_{Q}}\left(\Psi^{(l)}\left(\Psi^{(l)} \circ T^{j}\right)\right)\right) .
\end{aligned}
$$

By the ergodic theorem, $P$-a.s.,

$$
\lim _{k \rightarrow \infty} \frac{t_{\omega}(k)}{k}=\bar{l},
$$

and so

$$
\lim _{k \rightarrow \infty} \mu^{\omega}\left\{\xi \in \Xi^{\omega}: \frac{1}{\sqrt{t_{\omega}(k)}} \sum_{i=0}^{k}\left(\psi^{(l)} \circ \tau^{i}\right)_{\omega}(\xi) \leq a\right\}=\frac{1}{\sigma \sqrt{2 \pi}} \int_{-\infty}^{a} e^{-\frac{x^{2}}{2 \sigma^{2}}} d x
$$


Theorem 2.8 will follow if I show that for any $\varepsilon>0$ and $P$-a.a. $\omega$,

$$
\lim _{k \rightarrow \infty} \mu^{\omega}\left\{\xi \in \Xi^{\omega}: \frac{1}{\sqrt{k}}\left|\sum_{i=0}^{k}\left(\psi^{(l)} \circ \tau^{i}\right)_{\omega}(\xi)-\sum_{i=0}^{n_{\omega}\left(\xi, t_{\omega}(k)\right)}\left(\psi^{(l)} \circ \tau^{i}\right)_{\omega}(\xi)\right|>\varepsilon\right\}=0 .
$$

Actually, it suffices to obtain (7.11) for $P_{Q}-$ a.a. $\omega$, since $n^{-1 / 2} \sum_{i=0}^{k_{1}(\omega)}\left(\psi^{(l)} \circ \tau^{i}\right)_{\omega} \rightarrow 0$ as $n \rightarrow \infty$. Taking into account (7.3), this will hold true if, $P_{Q}-$ a.s.,

$$
\lim _{k \rightarrow \infty} \sum_{-k^{\frac{1}{2}+\alpha} \leq j \leq k^{\frac{1}{2}+\alpha}} \mu^{\omega}\left\{\xi \in \Xi^{\omega}: \frac{1}{\sqrt{k}}\left|L_{k, j}(\xi, \omega)\right|>\varepsilon\right\}=0
$$

where $L_{k, j}(\xi, \omega)=r_{|j|} \circ \tau^{k+j}(\xi, \omega)$ and $r_{n}(\xi, \omega)=\sum_{i=0}^{n}\left(\psi^{(l)} \circ \tau^{i}\right)_{\omega}(\xi)$. It suffices to show that there exists $C_{2}$ such that for any $n=1,2, \ldots$,

$$
E_{\mu_{Q}} r_{n}^{8} \leq C_{2} n^{4} .
$$

Indeed, by the Chebyshev inequality the sum in (7.12) does not exceed

$$
M_{k}(\omega) \stackrel{\text { def }}{=} \varepsilon^{-8} k^{-4} \sum_{-k^{\frac{1}{2}+\alpha} \leq j \leq k^{\frac{1}{2}+\alpha}} E_{\mu^{\theta^{k+j} \omega}}\left(r_{|j|}^{8}\right)_{\theta^{k+j} \omega} .
$$

If (7.13) holds true, then

$$
E_{P_{Q}} M_{k} \leq 2 C_{2} \varepsilon^{-8} k^{-\frac{3}{2}+5 \alpha} .
$$

If $\alpha<\frac{1}{10}$ then the right hand side of (7.15) is a $k$-th term of a converging series, and so employing the Borel-Cantelli lemma I conclude that $P_{Q}$-a.s. $\lim _{k \rightarrow \infty} M_{k}=0$, which proves (7.12).

In order to establish (7.13) I observe that

$$
\left(E_{\mu_{Q}} r_{n}^{8}\right)^{1 / 8} \leq\left(E_{\mu_{Q}} V_{n}^{8}\right)^{1 / 8}+\left(E_{\mu_{Q}}\left(R^{(l)}\right)^{8}\right)^{1 / 8},
$$

where $V_{n}=\sum_{j=0}^{N_{n}(\omega)-1} \Psi^{(l)} \circ T^{j}(\xi, \omega)$ with $N_{n}(\omega)$ and $R^{(l)}(\xi, \omega)$ being the same as in (3.1) and (2.32), respectively. I have to estimate only the first term in the right hand side of (7.16), since the last term there is bounded by (2.32). Since $N_{n}(\omega) \leq n$, then

$$
E_{\mu_{Q}} V_{n}^{8} \leq 8 ! \sum_{0 \leq i_{1} \leq i_{2} \leq \cdots \leq i_{8}} E_{P_{Q}}\left|E_{\mu^{\omega}} \prod_{j=1}^{8} \Psi^{(l)} \circ T^{i_{j}}\right|,
$$

and so one obtains (7.13) from the following result.

7.1. Lemma. Let $\Psi=\Psi_{\omega}(\xi)$ be a measurable function on $\Xi$ such that $\Psi_{\omega}$ is $\mathcal{F}_{0, \infty}^{\omega}-$ measurable function on $\Xi^{\omega}$ with $E_{\mu^{\omega}} \Psi_{\omega}=0$. Set

$$
D_{n}^{(m)}=\left(E_{\mu^{\omega}}\left(\Psi_{\omega}-E_{\mu^{\omega}}\left(\Psi_{\omega} \mid \mathcal{F}_{0, n}^{\omega}\right)\right)^{2 m}\right)^{\frac{1}{2 m}}
$$

and $\beta_{j}^{(m)}=\left(E_{P_{Q}}\left(D_{k_{j}}^{(m)}\right)^{2 m}\right)^{\frac{1}{2 m}}$. Suppose that

$$
\begin{gathered}
E_{\mu_{Q}} \Psi^{2 m}<\infty, \\
\sum_{j=1}^{\infty} j^{m} \phi_{j}^{\frac{1}{2 m}}<\infty,
\end{gathered}
$$


and

$$
\sum_{j=1}^{\infty} j^{m} \beta_{j}<\infty
$$

Then there is a constant $C=C_{m}$ such that for any $n=1,2, \ldots$,

$$
\sum_{0 \leq i_{1} \leq \cdots \leq i_{2 m} \leq n} E_{P_{Q}}\left|E_{\mu^{\omega}} \prod_{j=1}^{2 m} \Psi \circ T^{i_{j}}\right| \leq C n^{m},
$$

and so

$$
E_{\mu_{Q}}\left(\sum_{i=0}^{n} \Psi \circ T^{i}\right)^{2 m} \leq 2 m ! C n^{m} .
$$

Proof. The argument resembles the proofs of Lemma 2.1 from [Kh], of Lemma 4 from $\S 20$ in [Bi], and of Theorem 2 from Section 1.4.1 in [Do] (see also Remark 4 there), and it proceeds in the following way. Let

$$
s=s_{i_{1}, \ldots, i_{2 m}}=\max _{1 \leq q<m}\left(i_{2 q+1}-i_{2 q}\right)=i_{2 r+1}-i_{2 r} .
$$

Then by (3.7) and the Cauchy-Schwarz inequality,

$$
\begin{aligned}
& I_{i_{1}, \ldots, i_{2 m}}^{\omega} \stackrel{\text { def }}{=} \mid E_{\mu^{\omega}}\left(\prod_{j=1}^{2 m} \Psi \circ T^{i_{j}}\right) \\
& -E_{\mu^{\omega}}\left(\prod_{j=1}^{2 r} E_{\mu \omega}\left(\Psi \circ T^{i_{j}} \mid \mathcal{F}_{0, k_{i_{2 r}+[s / 2]}}^{\omega}\right)\right) E_{\mu^{\omega}}\left(\prod_{j=2 r+1}^{2 m} \Psi \circ T^{i_{j}}\right) \mid \\
& \leq\left(2 \phi_{[s / 2]}^{1 / 2 m}+\sum_{u=1}^{2 r} D_{[s / 2]}^{(2 m)}\left(\Theta^{i_{u}} \omega\right)\left(E_{\mu^{\Theta^{i} u \omega}} \Psi_{\Theta^{i u} \omega}^{2 m}\right)^{-1 / 2 m}\right) \prod_{j=1}^{2 m}\left(E_{\mu^{\Theta^{i_{j}} \omega}} \Psi_{\Theta^{i_{j} \omega}}^{2 m}\right)^{1 / 2 m} .
\end{aligned}
$$

Furthermore, by the same reasons, for $t=i_{2 m}-i_{2 m-1}$ and each $v=1,2, \ldots, 2 m-2$,

$$
\begin{aligned}
& \left|E_{\mu \omega} \prod_{j=v}^{2 m} \Psi \circ T^{i_{j}}\right| \\
& \leq\left(2 \phi_{[t / 2]}^{1 / 2 m}+\sum_{u=v}^{2 m-1} D_{[t / 2]}^{(2 m)}\left(\Theta^{i_{u}} \omega\right)\left(E_{\mu^{\Theta^{i} u} \omega} \Psi_{\Theta^{i} u \omega}^{2 m}\right)^{-1 / 2 m}\right) \prod_{j=v}^{2 m}\left(E_{\mu^{\Theta^{i} j_{\omega}}} \Psi_{\Theta^{i_{j}} \omega}^{2 m}\right)^{1 / 2 m} .
\end{aligned}
$$

Using (7.24) with $v=1$ and $v=2 r+1$, it follows from (7.18), (7.23), and the Cauchy-Schwarz inequality that

$$
E_{P_{Q}} I_{i_{1}, \ldots, i_{2 m}} \leq 2 \phi_{\left[\frac{1}{2} \max (s, t)\right]}^{1 / 2 m} E_{\mu_{Q}} \Psi^{2 m}+2 m \beta_{\left[\frac{1}{2} \max (s, t)\right]}^{(2 m)}\left(E_{\mu_{Q}} \Psi^{2 m}\right)^{7 / 8},
$$

which by (7.19) and (7.20) yields that

$$
\sum_{0 \leq i_{1} \leq \ldots \leq i_{2 m} \leq n} E_{P_{Q}} I_{i_{1}, \ldots, i_{2 m}} \leq \tilde{C} n^{m}
$$


for some constant $\tilde{C}>0$ independent of $n$. Estimating

$$
E_{\mu^{\omega}}\left(\prod_{j=1}^{2 r} E_{\mu^{\omega}}\left(\Psi \circ T^{i_{j}} \mid \mathcal{F}_{0, k_{i_{2 r}+[s / 2]}}^{\omega}\right)\right) \text { and } E_{\mu^{\omega}}\left(\prod_{j=2 r+1}^{2 m} \Psi \circ T^{i_{j}}\right)
$$

similarly to (7.23)-(7.26) and continuing in the same way, one eventually arrives at (7.21).

\section{REFERENCES}

[Bi1] P. Billingsley, Convergence of Probability Measures, J.Wiley, New York, 1968. MR 38:1718

[Bi2] P. Billingsley, Probability and Measure, J.Wiley, New York, 1986. MR 87f:60001

[Bo] R. Bowen, Equilibrium States and the Ergodic Theory of Anosov Diffeomorphisms, Lect. Notes in Math. 470, Springer-Verlag, Berlin, 1975. MR 56:1364

[BG1] T. Bogenschutz and V. M. Gundlach, Symbolic dynamics for expanding random dynamical systems, Random \& Comp. Dyn. 1 (1992), 219-227. MR 93j:58042

[BG2] T. Bogenschutz and V. M. Gundlach, Ruelle's transfer operator for random subshifts of finite type, Ergod. Th. \& Dynam. Sys. 15 (1995), 413-447. MR 96m:58133

[Co] R. Cogburn, On the central limit theorem for Markov chains in random environments, Ann. Probab. 19 (1991), 587-604. MR 92h:60029

[CFS] I. P. Cornfeld, S. V. Fomin, and Ya. G. Sinai, Ergodic Theory, Springer-Verlag, Berlin, 1982. MR 87f:28019

[De] M. Denker, The central limit theorem for dynamical systems, Dynamical Systems and Ergodic Theory, Banach Center Publ., vol. 23, PWN-Polish Sci. Publ., Warszawa, 1989, pp. 33-62. MR 92d:28007

[Do] P. Doukhan, Mixing: Properties and Examples, Lecture Notes in Stat. 85, SpringerVerlag, New York, 1994. MR 96b:60090

[DP] M. Denker and W. Philipp, Approximation by Brownian motion for Gibbs measures and flows under a function, Ergod. Th. \& Dynam. Sys. 4 (1984), 541-552. MR 86g:28025

[Go] M. I. Gordin, The central limit theorem for stationary processes, Soviet Math. Dokl. 10 (1969), 1174-1176. MR 40:5012

[Gu] V. M. Gundlach, Thermodynamic formalism for random subshifts of finite type, Preprint, 1996.

[Ha] P. Hartman, Ordinary Differential Equations, J.Wiley, New York, 1964. MR 30:1270

$[\mathrm{HH}] \quad$ P. Hall and C. C. Heyde, Martingale Limit Theory and its Applications, Acad. Press, New York, 1980. MR 83a:60001

[HS] C. C. Heyde and D. J. Scott, Invariance principles for the law of the iterated logarithm for martingales and processes with stationary increments, Ann. Probab. 1 (1973), 428436. MR 50:5886

[IL] I.A.Ibragimov and Y.V.Linnik, Independent and Stationary Sequences of Random Variables, Wolters-Nordhoff, Groningen, 1971. MR 48:1287

[Kh] R.Z.Khasminskii, On stochastic processes defined by differential equations with a small parameter, Theory Probab. and its Appl. 11 (1966), 211-228. MR 34:3636

[Ki1] Y. Kifer, Equilibrium states for random expanding transformations, Random \& Comp. Dyn. 1 (1992), 1-31. MR 93i:58075

[Ki2] Y. Kifer, Limit theorems in averaging for dynamical systems, Ergod. Th. \& Dynam. Sys. 15 (1995), 1143-1172. MR 97e:58087

[Ki3] Y. Kifer, Perron-Frobenius theorem, large deviations, and random perturbations in random environments, Math. Zeitschrift 222 (1996), 677-698. MR 97f:60131

[Ki4] Y. Kifer, Fractal dimensions and random transformations, Trans. Amer. Math. Soc. 348 (1996), 2003-2038. MR 96i:28009

[KK] K. Khanin and Y. Kifer, Thermodynamic formalism for random transformations and statistical mechanics, in: Sinai's Moscow Seminar on Dynamical Systems (L.A.Bunimovich, B.M.Gurevich, Ya.B.Pesin, eds.), American Mathematical Society Translations (2) $\mathbf{1 7 1}$ (1995), 107-140. MR 96j:58136 
[Li] C. Liverani, Central limit theorem for deterministic systems, in: Proc. Int. Congr. in Dynam. Sys. (F.Ledrappier, J.Lewowicz, and S.Newhouse, eds.), Pitman Research Notes in Mathematics, Longman, Harlow, 1996.

[Or] S. Orey, Markov chains with stochastically stationary transition probabilities, Ann. Probab. 19 (1991), 907-928. MR 92i:60065

[PS] W. Philipp and W. F. Stout, Almost sure invariance principles for sums of weakly dependent random variables, Memoirs of Amer. Math. Soc. 161, Amer. Math. Soc., Providence, 1975. MR 55:6570

$[\mathrm{Ru}] \quad$ B.-Z. Rubshtein, A central limit theorem for conditional distributions, in: Convergence in Ergodic Theory and Probability (Bergelson, March, Rosenblatt, eds.), Walter de Gruyter, Berlin, 1996. CMP 97:02

[Se] T. Seppäläinen, Large deviations for Markov chains with random transitions, Ann. Probab. 22 (1994), 713-748. MR 95j:60044

[Sh] A.N.Shiryaev, Probability, Springer-Verlag, New York, 1984. MR 85a:60007

[VR] V.A.Volkonskii and Yu.A.Rozanov, Some limit theorems for random functions I, Th. Probab. Appl. 4 (1959), 178-197. MR 22:12586; MR 21:4477

Institute of Mathematics, Hebrew University of Jerusalem, Givat Ram, Jerusalem 91904, ISRAEL

E-mail address: kifer@math.huji.ac.il 